\title{
ZnO:Al Doping Level and Hydrogen Growth Ambient Effects on CIGS \\ Solar Cell Performance
}

Joel N. Duenow

David M. Wood

Brian Egaas

Colorado School of Mines

Golden, Colorado, USA
Timothy A. Gessert

Rommel Noufi

Timothy J. Coutts

National Renewable Energy Laboratory

Golden, Colorado, USA

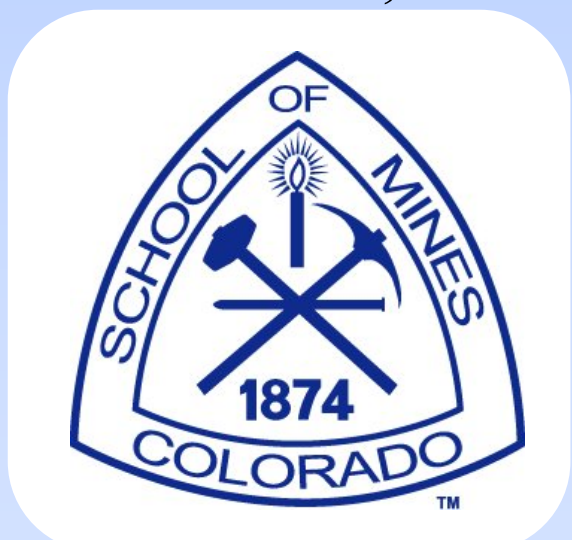

33rd IEEE Photovoltaic Specialists Conference

May 12, 2008

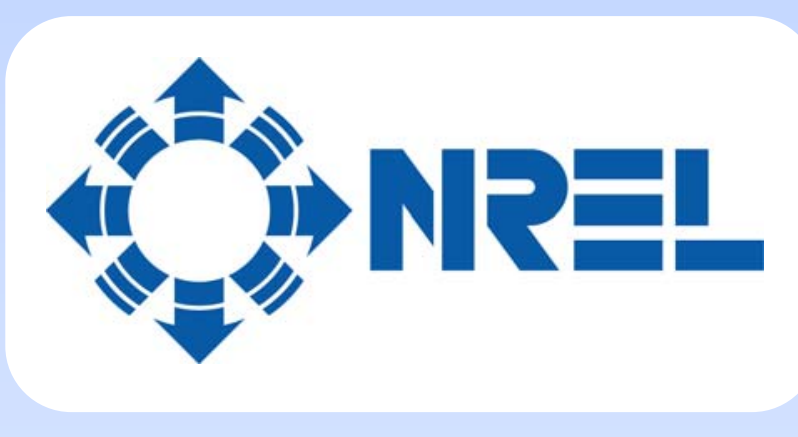

NREL/PR-520-43257

Presented at the 33rd IEEE Photovoltaic Specialist Conference held May 11-16, 2008 in San Diego, California

This work was supported by DOE contract DE-AC36-99G010337 and NREL subcontract KXEA-3-33607-24 


\section{Modeled TCO Absorptance}

\section{Varying carrier conc.}

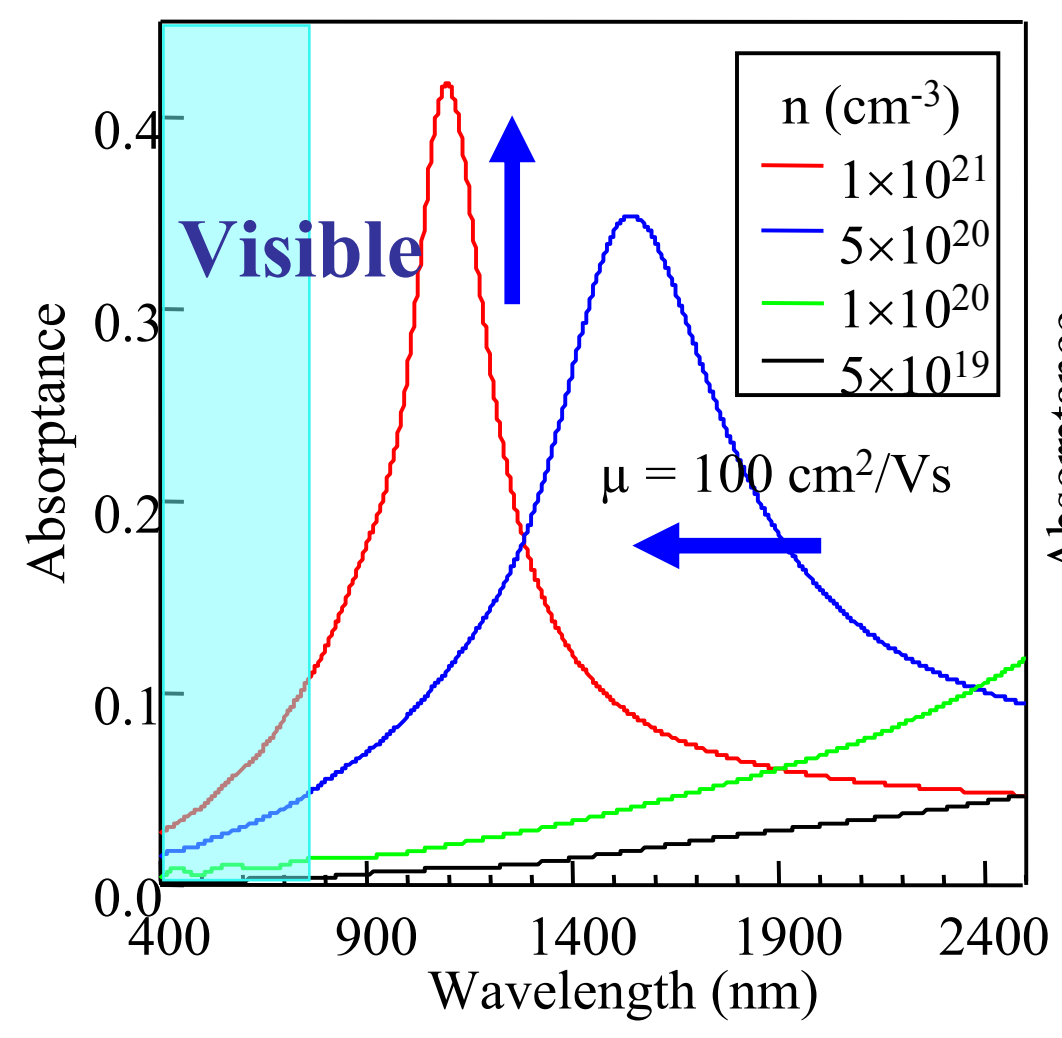

$\mathrm{A}=1-\mathrm{T}-\mathrm{R}$

$\sigma=q n \mu$

T. Coutts et al., MRS Bulletin 25, 58 (2000)
Varying mobility

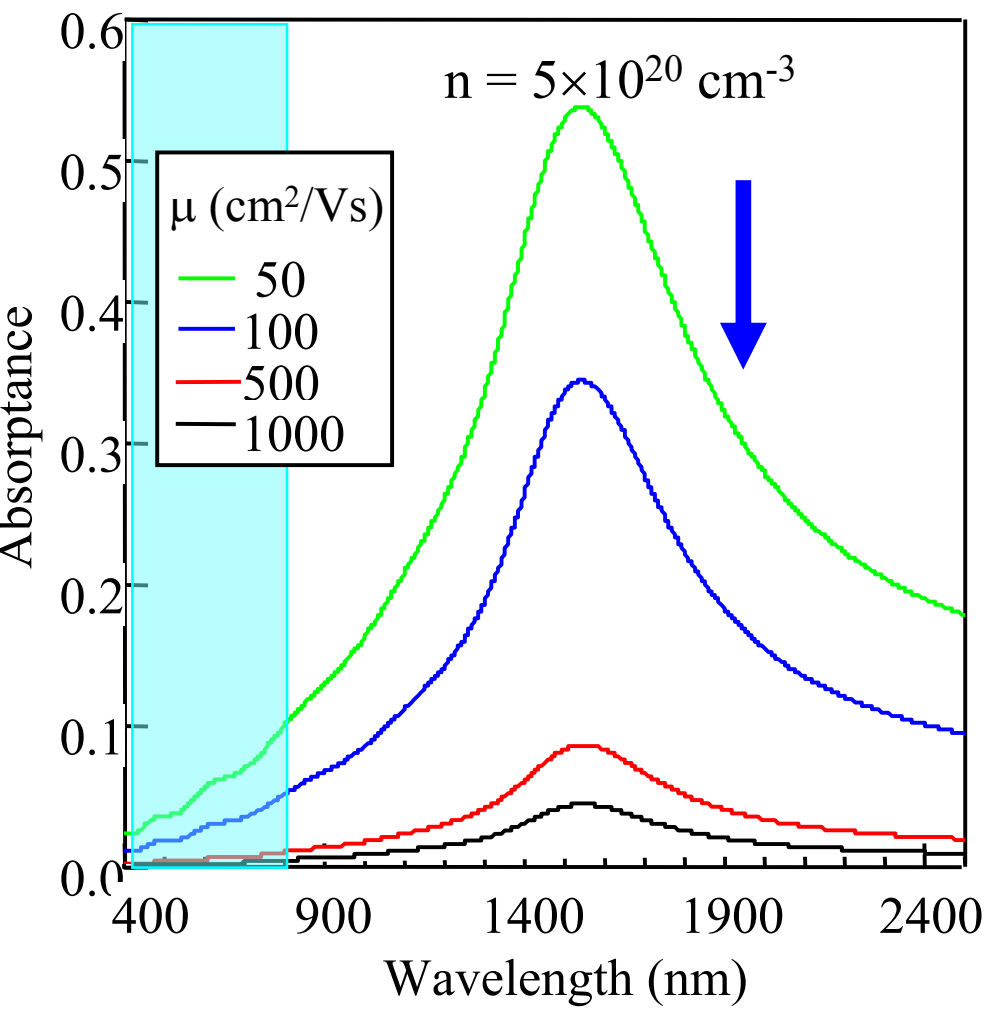

$$
\omega_{p}=\frac{2 \pi}{\lambda_{p}}=\sqrt{\frac{4 \pi n e^{2}}{m^{*}}}
$$

Best optical properties by increasing mobility rather than carrier concentration 


\section{Investigations in this study}

\section{ZnO:Al Studies}

- ZnO:Al with 2.0 wt. $\% \mathrm{Al}_{2} \mathrm{O}_{3}$ commonly used, but limits carrier mobility

- We investigate lightly-doped $\mathrm{ZnO}$ :Al grown using small amounts of $\mathrm{H}_{2}$ in the Ar sputtering ambient

$$
\text { - 0.05, 0.1, 0.2, 0.5, 1.0, } 2.0 \text { wt. } \% \mathrm{Al}_{2} \mathrm{O}_{3}
$$

\section{CIGS PV Device Studies}

Compare CIGS PV devices with lightly-doped and standard $\mathrm{ZnO}: \mathrm{Al}$ (0.1 wt.\% $\mathrm{Al}_{2} \mathrm{O}_{3}$ vs. 2.0 wt. $\% \mathrm{Al}_{2} \mathrm{O}_{3}$ ) 


\section{Film Growth}

Unifilm PVD-300

Sputter Deposition
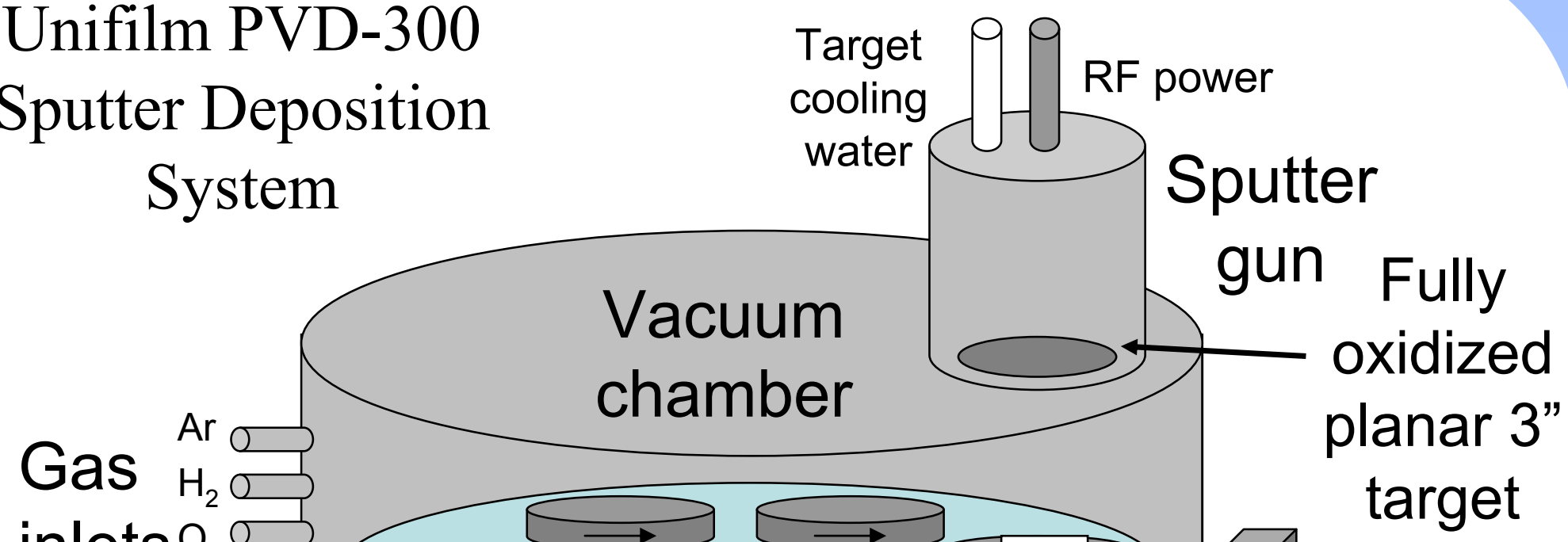

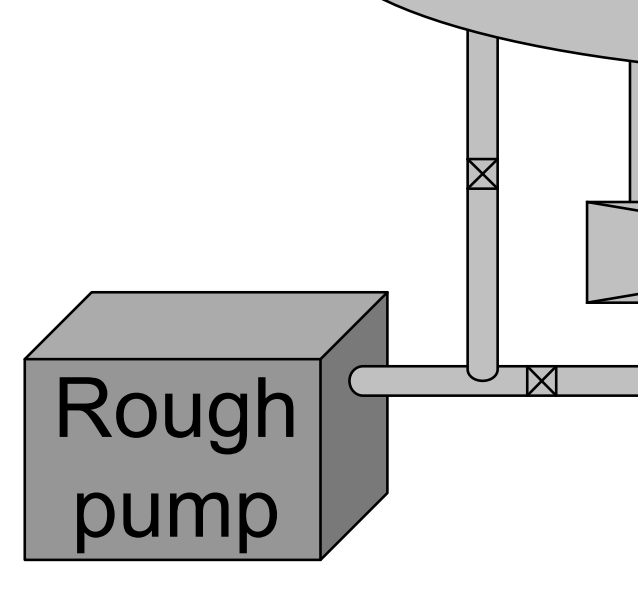
inlets $\mathrm{O}_{2} \sigma$

Vacuum chamber

Gas Ar $\mathrm{Ar}^{\circ}$
$\mathrm{H}_{2} \sigma$
$\mathrm{O}_{2} \sigma$
Corning 7059 or 1737 Cryogenic gauge Substrate 


\section{Electrical Data - Ambient Studies}

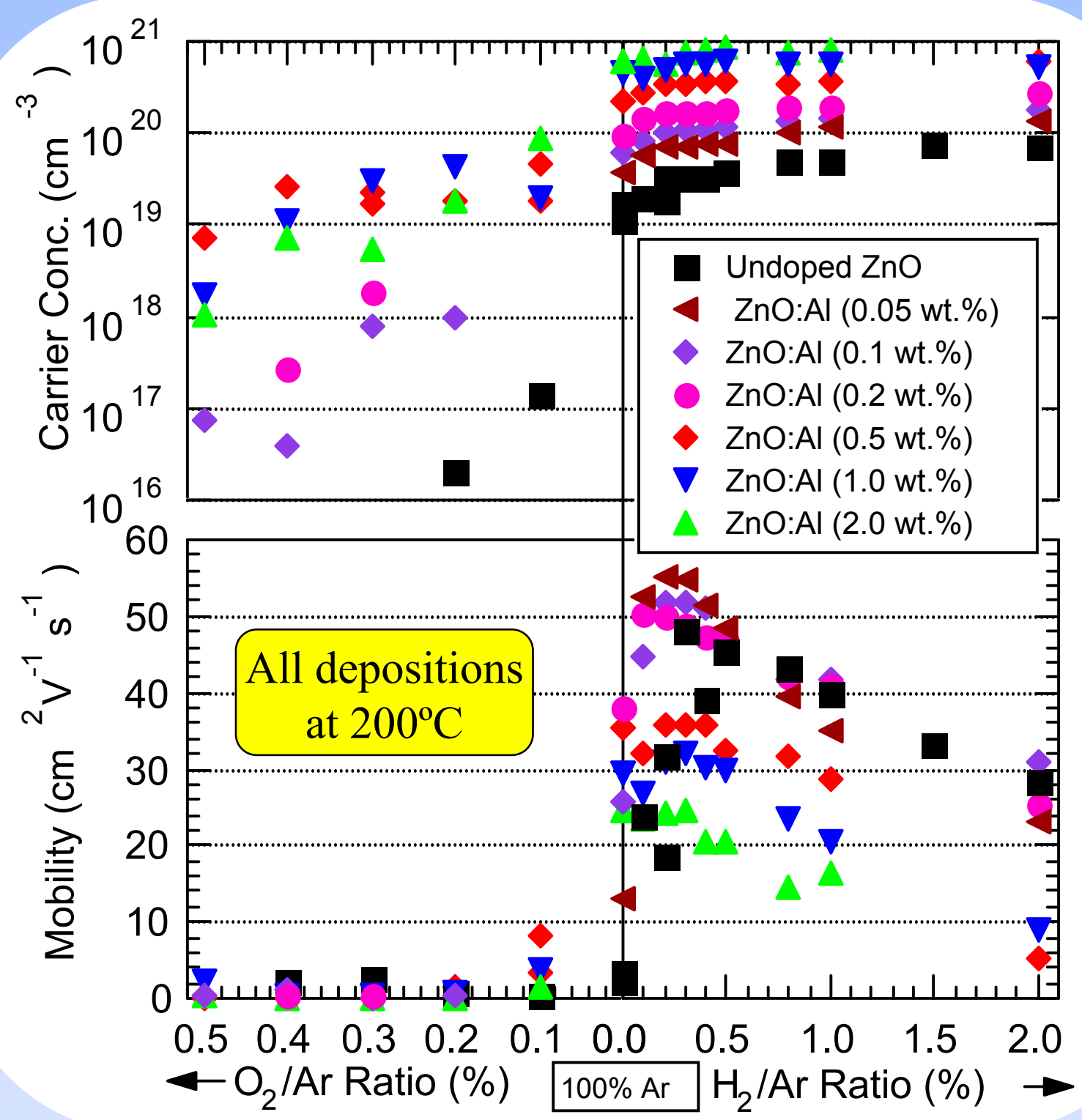

- Adding $\mathrm{O}_{2}$ sharply decreases both carrier concentration and mobility

- Adding $\mathrm{H}_{2}$ in limited amount is beneficial to both
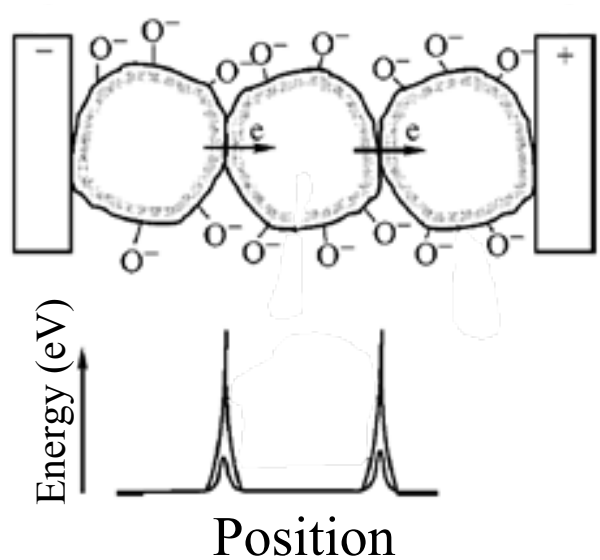


\section{Electrical Data - Substrate Temp. Series $100 \% \mathrm{Ar}$ and $0.3 \% \mathrm{H}_{2} / \mathrm{Ar}, 0.2$ wt. $\% \mathrm{Al}_{2} \mathrm{O}_{3}$}
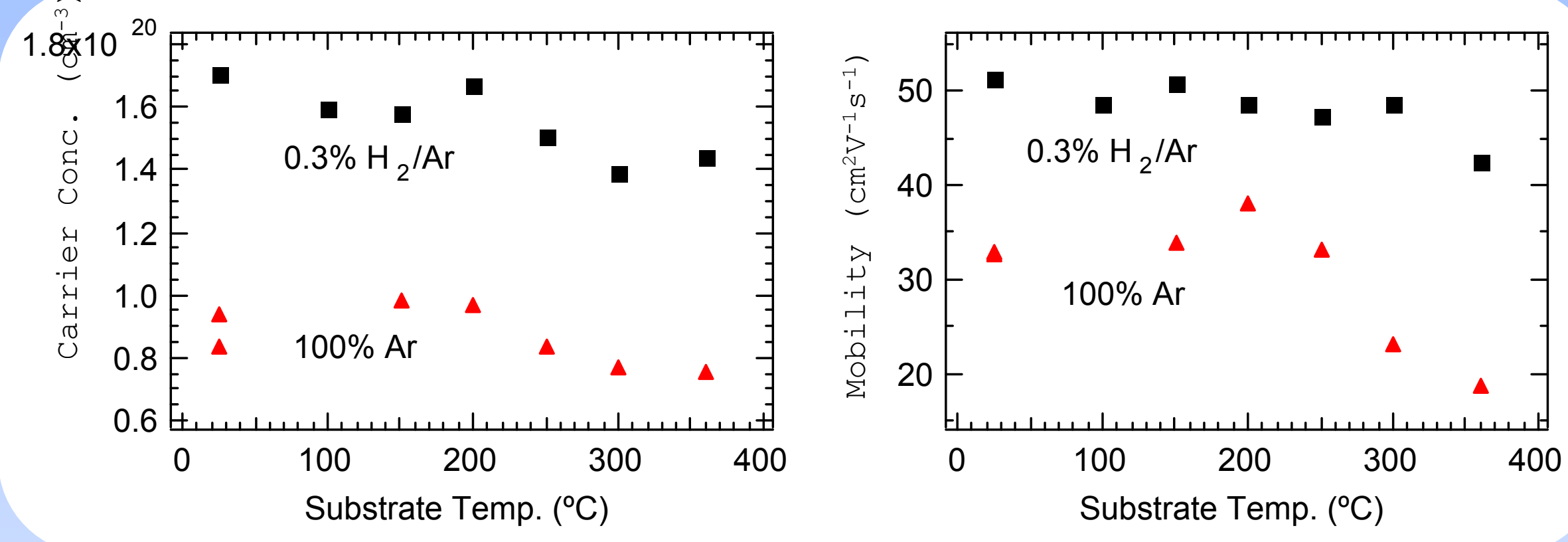

- $100 \%$ Ar peaks at $\sim 150-200^{\circ} \mathrm{C}$

- Slight monotonic decrease for $0.3 \% \mathrm{H}_{2} / \mathrm{Ar}$

- Tolerance for higher substrate T with $\mathrm{H}_{2}$ added 


\section{Optical} Data
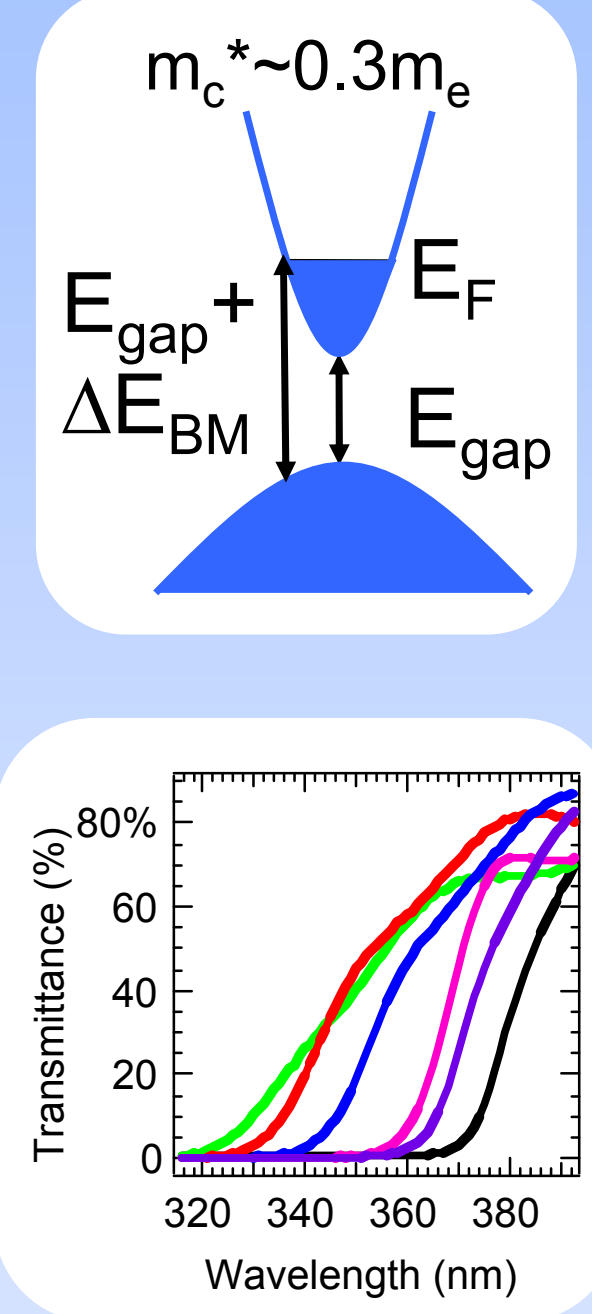

\begin{tabular}{|cccccc|}
\hline \multicolumn{7}{|c|}{ Best optical properties for ZnO-based films, substrate temp. $200^{\circ} \mathrm{C}$} \\
Thick. (nm) & $\mathrm{n}(\mathrm{cm}$ & $\left.{ }^{-3}\right)$ & $\mu\left(\mathrm{cm}^{2} / \mathrm{Ns}\right)$ & $\rho(\mathrm{z} \mathrm{cm})$ \\
\hline Undoped ZnO & 390 & $3.3 \times 10$ & 19 & 48 & $4.0 \times 10^{-3}$ \\
\hline ZnO:Al (0.1 wt.\%) & 370 & $1.1 \times 10$ & 20 & 52 & $1.1 \times 10^{-3}$ \\
\hline ZnO:Al (0.2 wt.\%) & 420 & $1.7 \times 10$ & 20 & 49 & $7.7 \times 10^{-4}$ \\
\hline ZnO:Al (0.5 wt.\%) & 410 & $3.4 \times 10$ & 20 & 36 & $5.1 \times 10^{-4}$ \\
\hline ZnO:Al (1.0 wt.\%) & 490 & $5.5 \times 10$ & 20 & 32 & $3.6 \times 10^{-4}$ \\
ZnO:Al (2.0 wt.\%) & 470 & $5.9 \times 10$ & 20 & 25 & $4.3 \times 10^{-4}$ \\
\hline
\end{tabular}

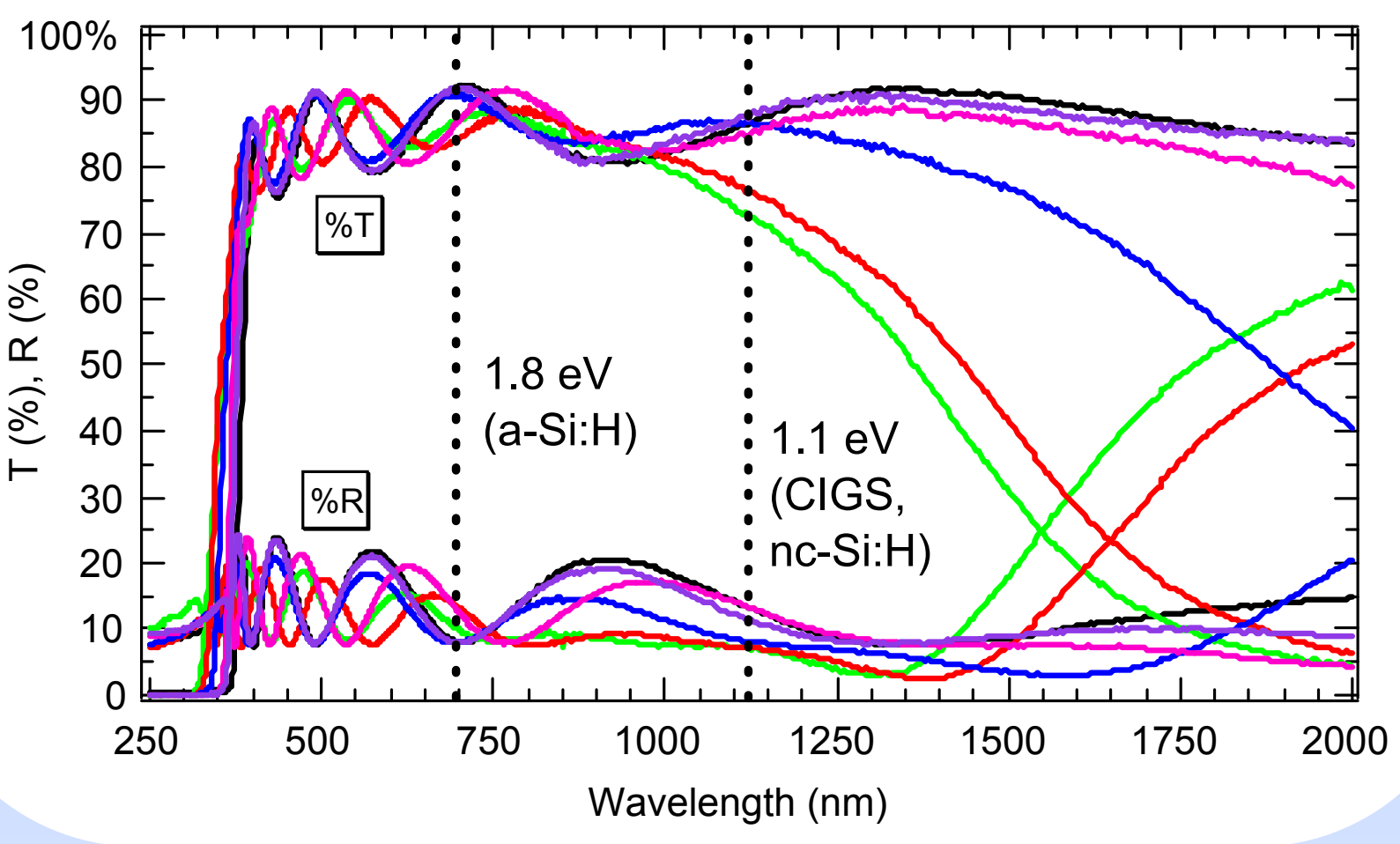

-Burstein-Moss shift observed -Free-carrier absorption in infrared 


\section{CIGS PV Device Studies}

Control:

$$
2.0 \text { wt. } \% \mathrm{Al}_{2} \mathrm{O}_{3}
$$

- CdS by chemical bath deposition

- 100 nm IZO, 120 nm ZnO:Al

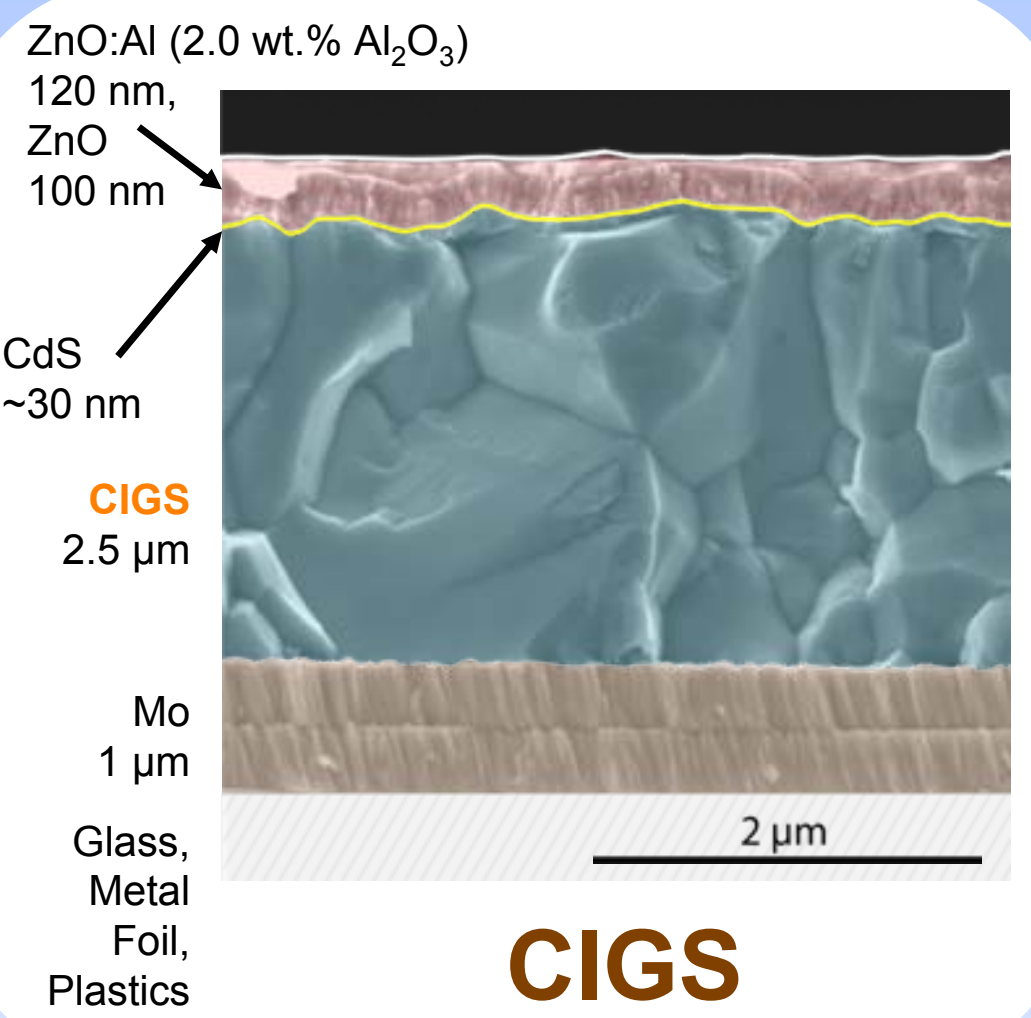

Test:

0.1 wt. $\% \mathrm{Al}_{2} \mathrm{O}_{3}$

- CdS/ZnS ( 20/30 nm)

- $100 \mathrm{~nm}$ IZO, $120 \mathrm{~nm} \mathrm{ZnO}: \mathrm{Al}$

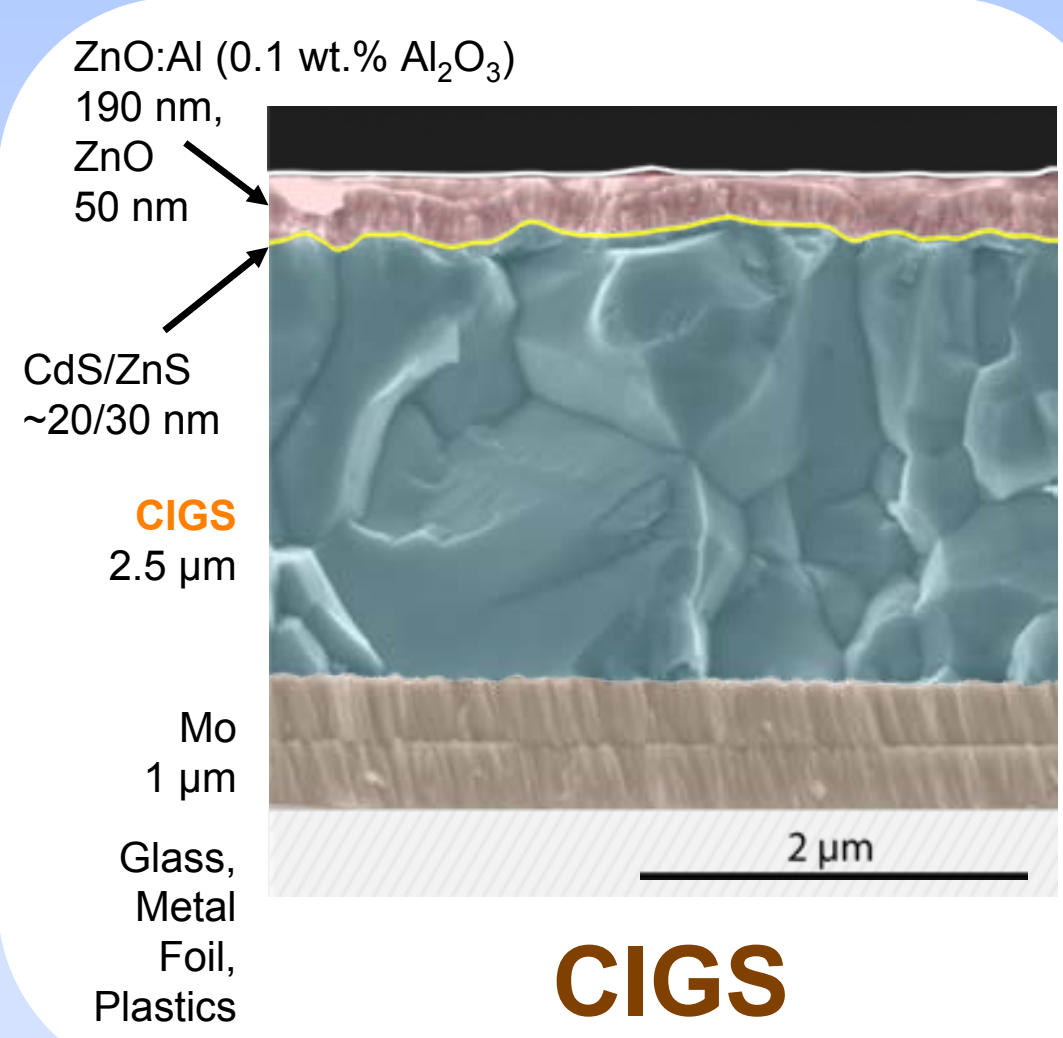




\section{CIGS PV Device Studies - 2}

- Efficiency, FF, $\mathrm{V}_{\mathrm{OC}}$, $\mathrm{J}_{\mathrm{SC}}$ compare favorably with control sample

- QE: Difference at low wavelengths due to $\mathrm{CdS}$ vs. $\mathrm{CdS} / \mathrm{ZnS}$

- At higher wavelengths, $\mathrm{QE}$ of $0.1 \% \mathrm{Al}_{2} \mathrm{O}_{3}$ cell rivals $19.5 \% \mathrm{WR}$ cell
Comparing $\mathrm{ZnO}$ :Al doping levels on CIGS

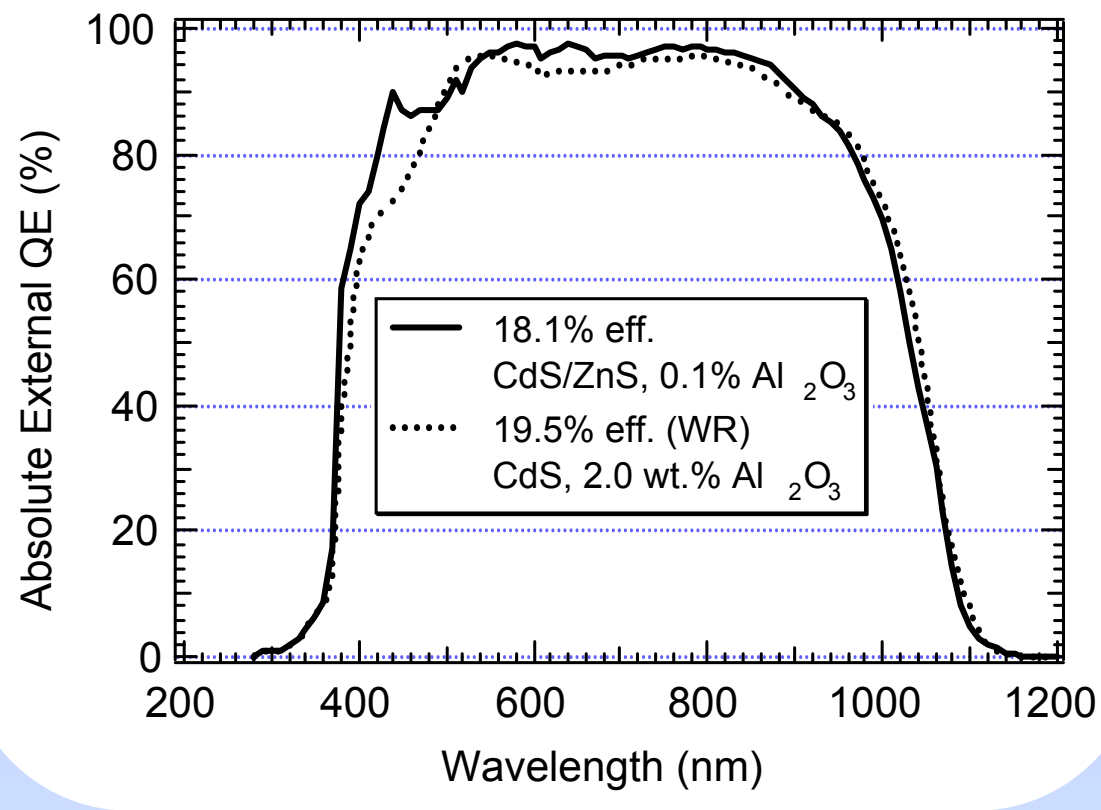

\begin{tabular}{|c|c|c|c|c|c|}
\hline $\begin{array}{c}\mathrm{Al}_{2} \mathrm{O}_{3} \text { Content } \\
(\text { wt. } \%)\end{array}$ & Treatment & Efficiency (\%) & Fill Factor (\%) & $\begin{array}{c}\text { Open-curcuit } \\
\text { voltage }(\mathrm{mV})\end{array}$ & $\begin{array}{c}\text { Short-circuit } \\
\text { current }\left(\mathrm{mA} / \mathrm{cm}^{2}\right)\end{array}$ \\
\hline 0.1 & $\mathrm{CdS} / \mathrm{ZnS}$ & 18.1 & 76.2 & 671 & 35.4 \\
\hline 2.0 & $\mathrm{CdS}$ & 18.1 & 79.1 & 666 & 34.4 \\
\hline
\end{tabular}




\section{Conclusions}

- Lightly-doped $\mathrm{ZnO}$ (grown in $\mathrm{H}_{2}$ ) can substitute for the standard 2.0 wt. $\% \mathrm{Al}_{2} \mathrm{O}_{3}$

- increased carrier mobility

- increased near-IR transmittance

- Addition of $\mathrm{H}_{2}$ enables best mobility and carrier concentration for $\mathrm{ZnO}$ :Al using room $\mathrm{T}$ deposition and increased tolerance for higher $\mathrm{T}$

- In initial CIGS PV device studies:

- Efficiency, FF, $\mathrm{V}_{\mathrm{OC}}, \mathrm{J}_{\mathrm{SC}}$ compare favorably with control

- QE comparable to former WR cell at higher wavelengths 
11 


\section{All CIGS PV Device Results}
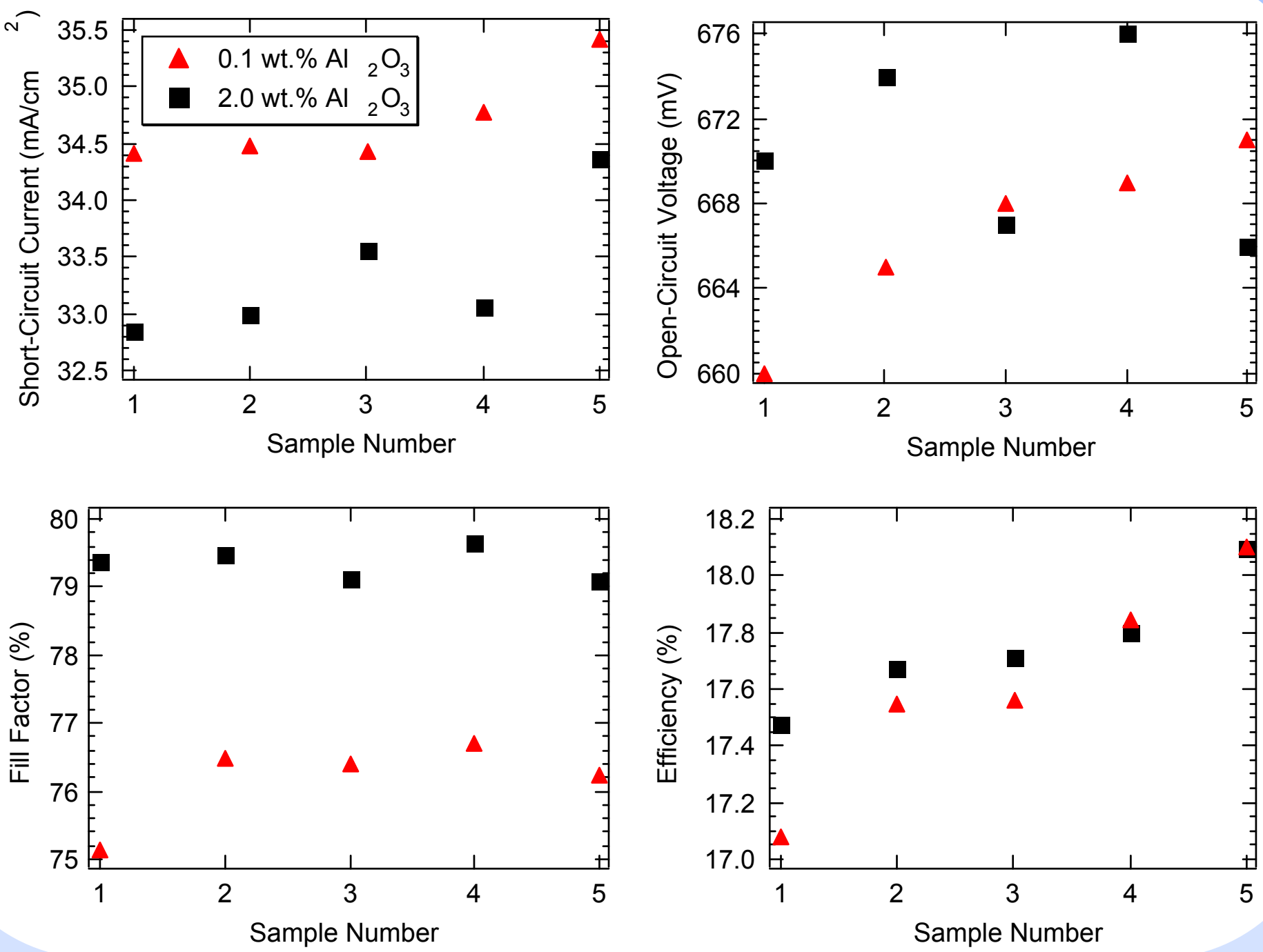


\section{Resistivity vs. $\mathrm{O}_{2} / \mathrm{Ar}$ and $\mathrm{H}_{2} /$ Ar Ratios}

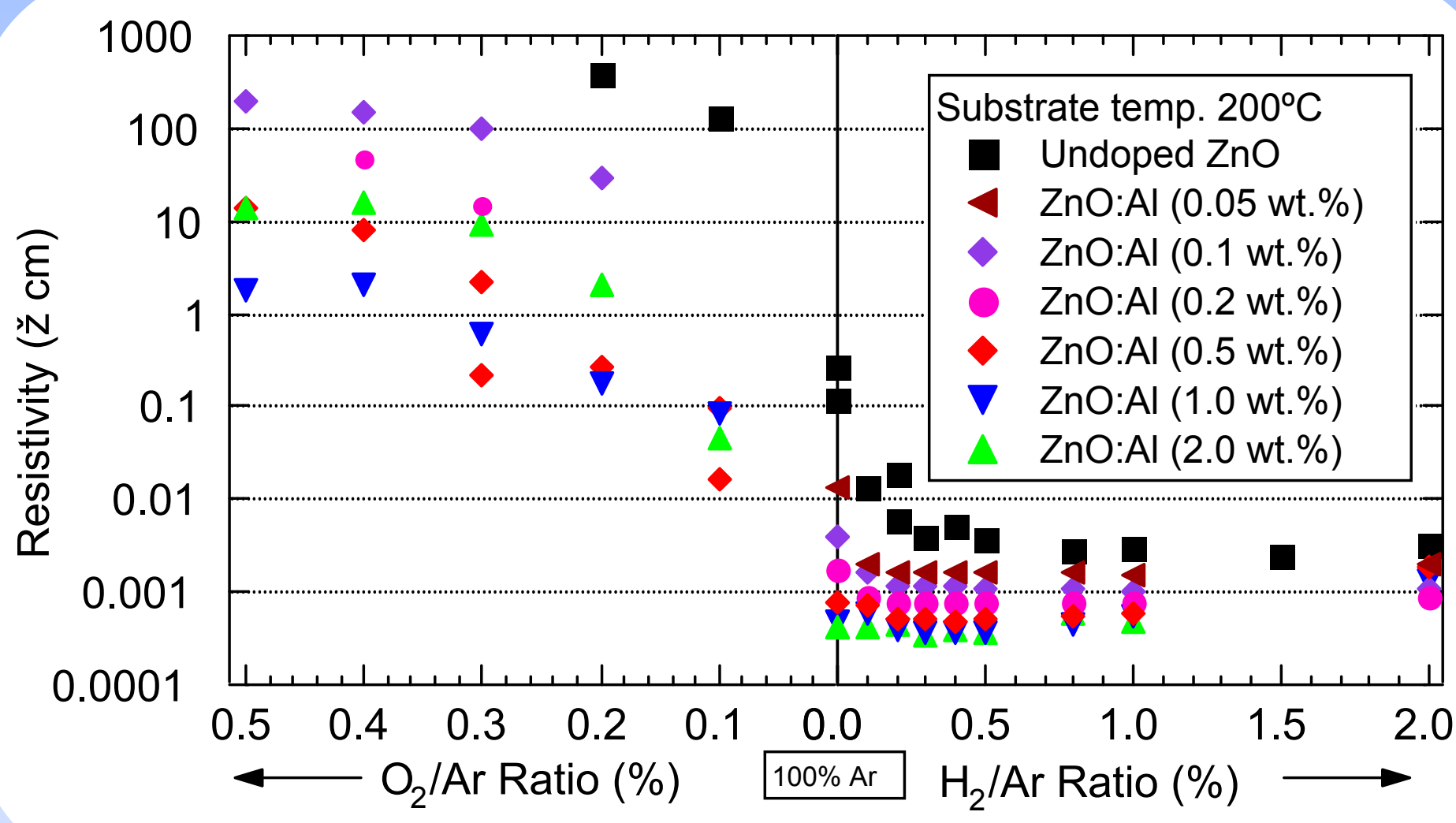




\section{Electrical Properties vs. Substrate Temp.}
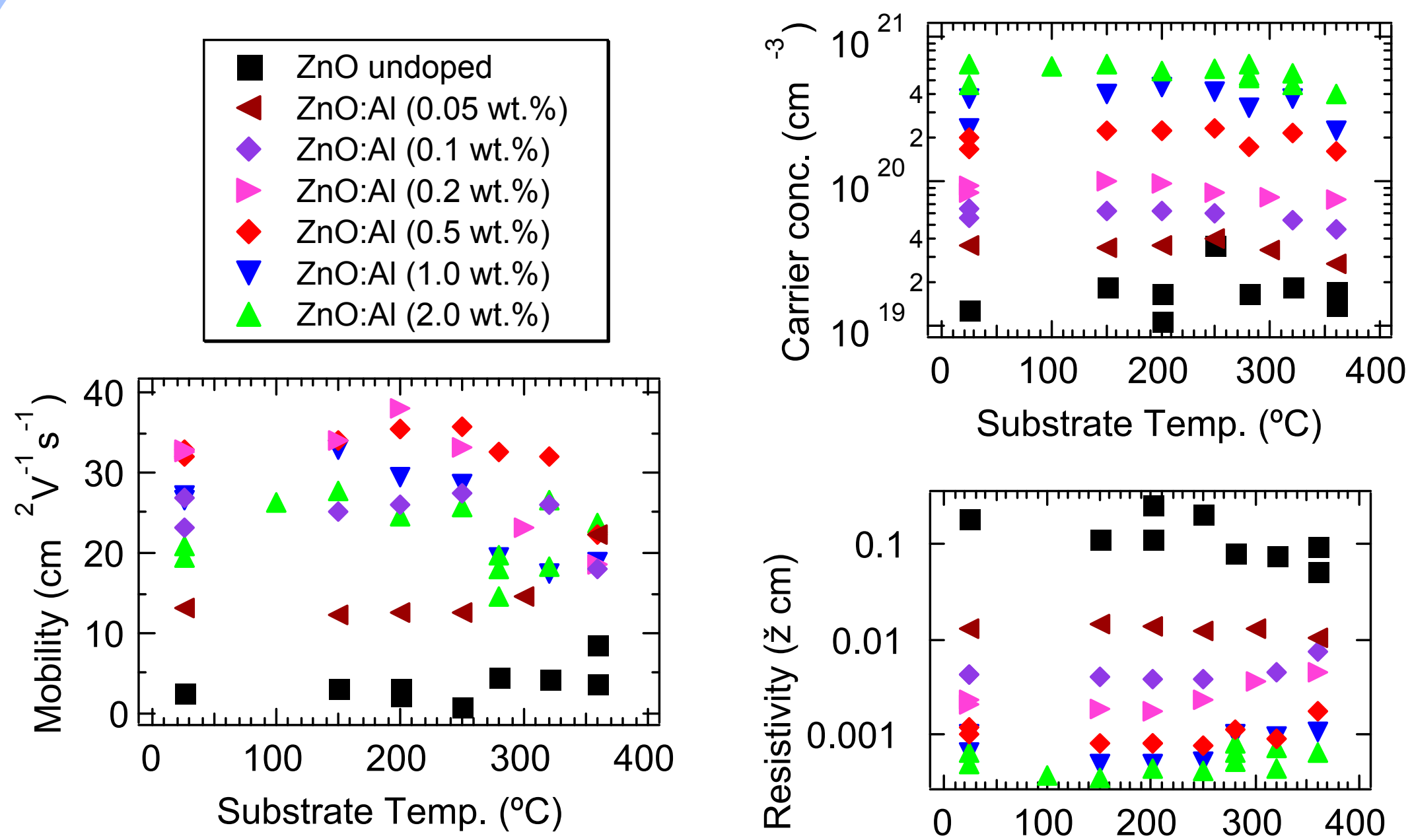

All films grown in 100\% $\mathrm{Ar}$

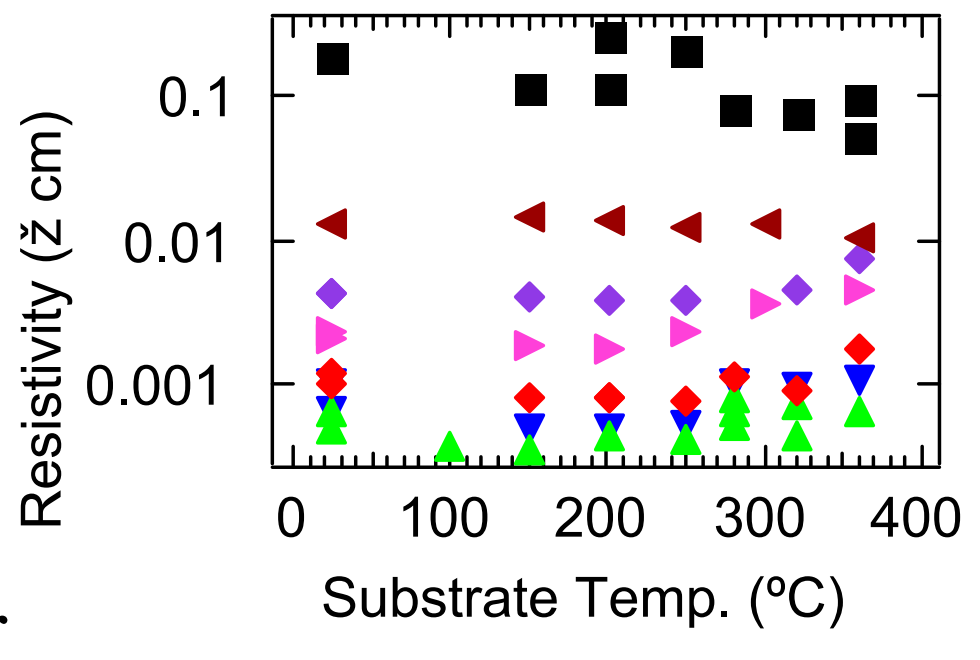




\section{Mobility ( $\mu$ ) vs. Carrier Concentration (n)}

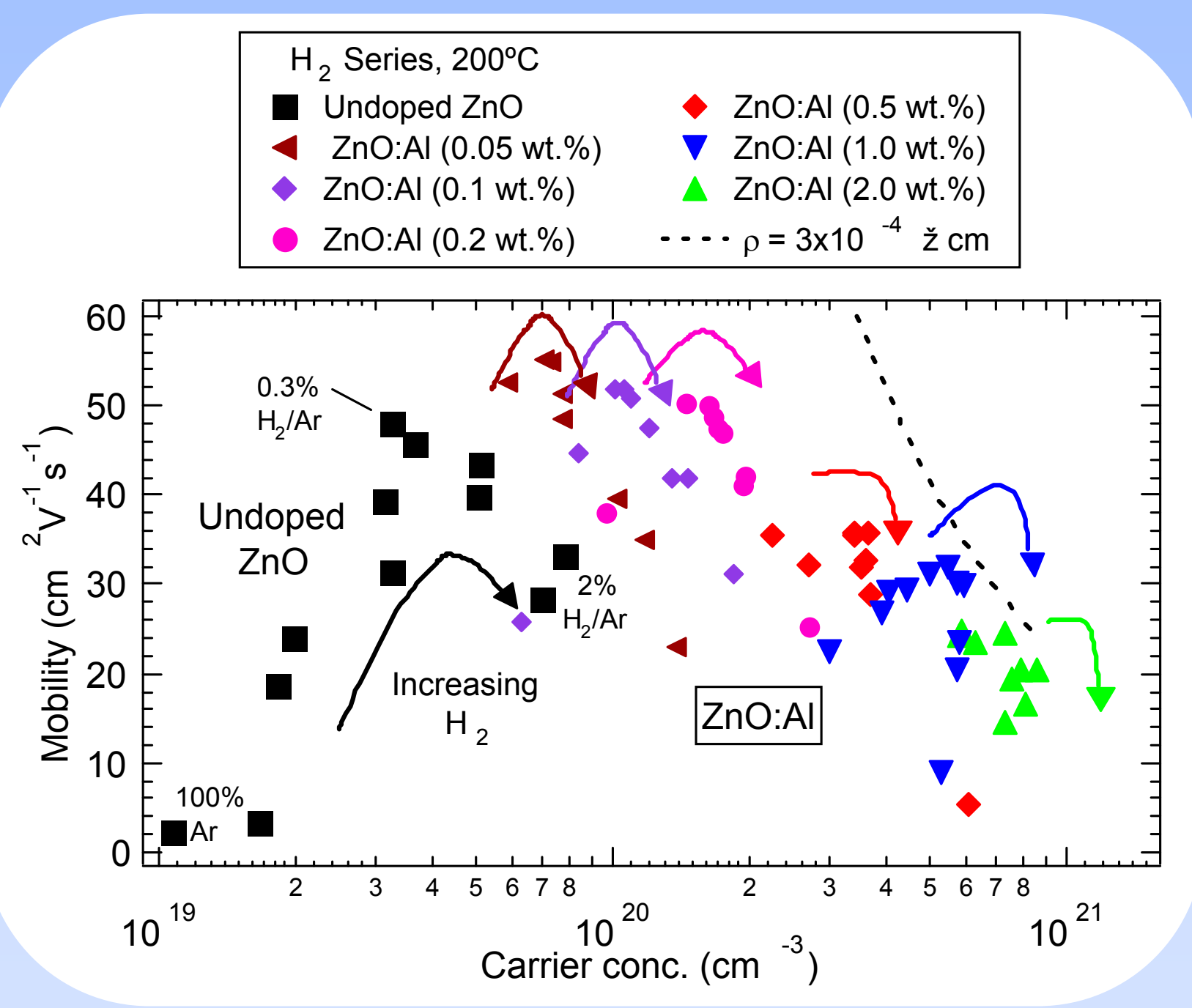

Undoped $\mathrm{ZnO}$

- Passivation of defects by $\mathrm{H}$

$\mathrm{ZnO}: \mathrm{Al}$

- Activation of dopant with $\mathrm{H}$

- Ionized impurity scattering

$\mathrm{H}_{2}$ : Filling sites (e.g. on grain boundaries) on which dopant atoms would not contribute carriers? 


\section{Absorptance vs. Wavelength}

\begin{tabular}{|c|c|c|c|}
\hline \multicolumn{4}{|c|}{ Best optical properties for $\mathrm{ZnO}$-based films, $200^{\circ} \mathrm{C}$} \\
\hline Thic & kness $(\AA)$ & $\mathrm{n}\left(\mathrm{cm}^{-3}\right)$ & $\mu\left(\mathrm{cm}^{2} \mathrm{~V}^{-1} \mathrm{~s}^{-1}\right)$ \\
\hline $\mathrm{ZnO}$ & 3900 & $3.3 \times 10^{19}$ & 48 \\
\hline $\mathrm{ZnO}: \mathrm{Al}(0.1$ wt. \%) & 3700 & $1.1 \times 10^{20}$ & 52 \\
\hline $\mathrm{ZnO}: \mathrm{Al}(0.2$ wt. \%) & 4200 & $1.7 \times 10^{20}$ & 49 \\
\hline $\mathrm{ZnO}: \mathrm{Al}(0.5 \mathrm{wt} . \%)$ & 4100 & $3.4 \times 10^{20}$ & 36 \\
\hline ZnO:Al (1 wt.\%) & 4900 & $5.5 \times 10^{20}$ & 32 \\
\hline ZnO:Al (2 wt.\%) & 4700 & $5.9 \times 10^{20}$ & 25 \\
\hline
\end{tabular}

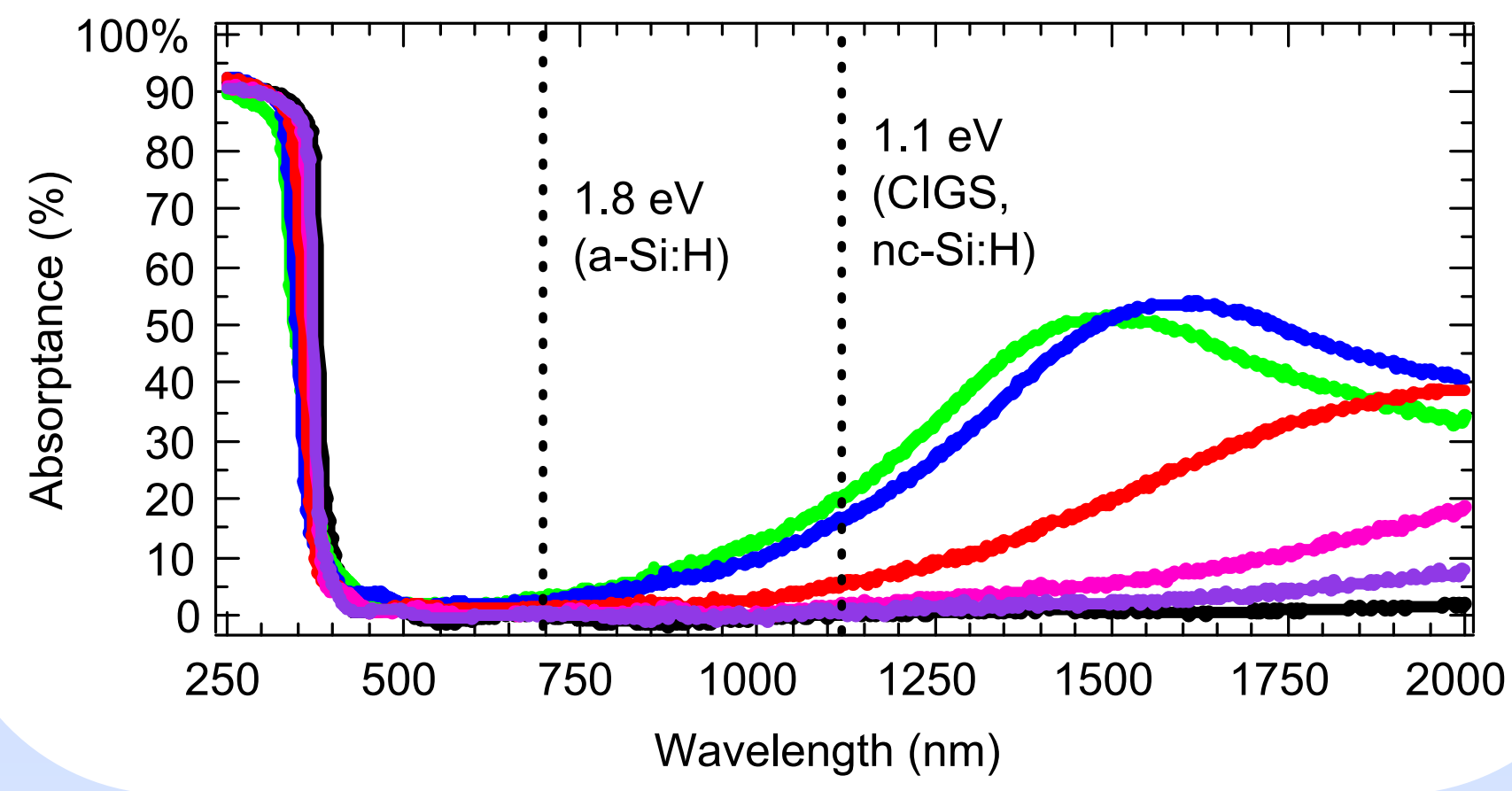




\section{To what extent is $\mathrm{H}_{2}$ incorporated}

in films?

- SIMS measurements show $\sim 10^{21} \mathrm{~cm}^{-3} \mathrm{H}$ conc.

- But carrier conc. is $\sim 10^{19} \mathrm{~cm}^{-3}$, so most $\mathrm{H}$ not ionized

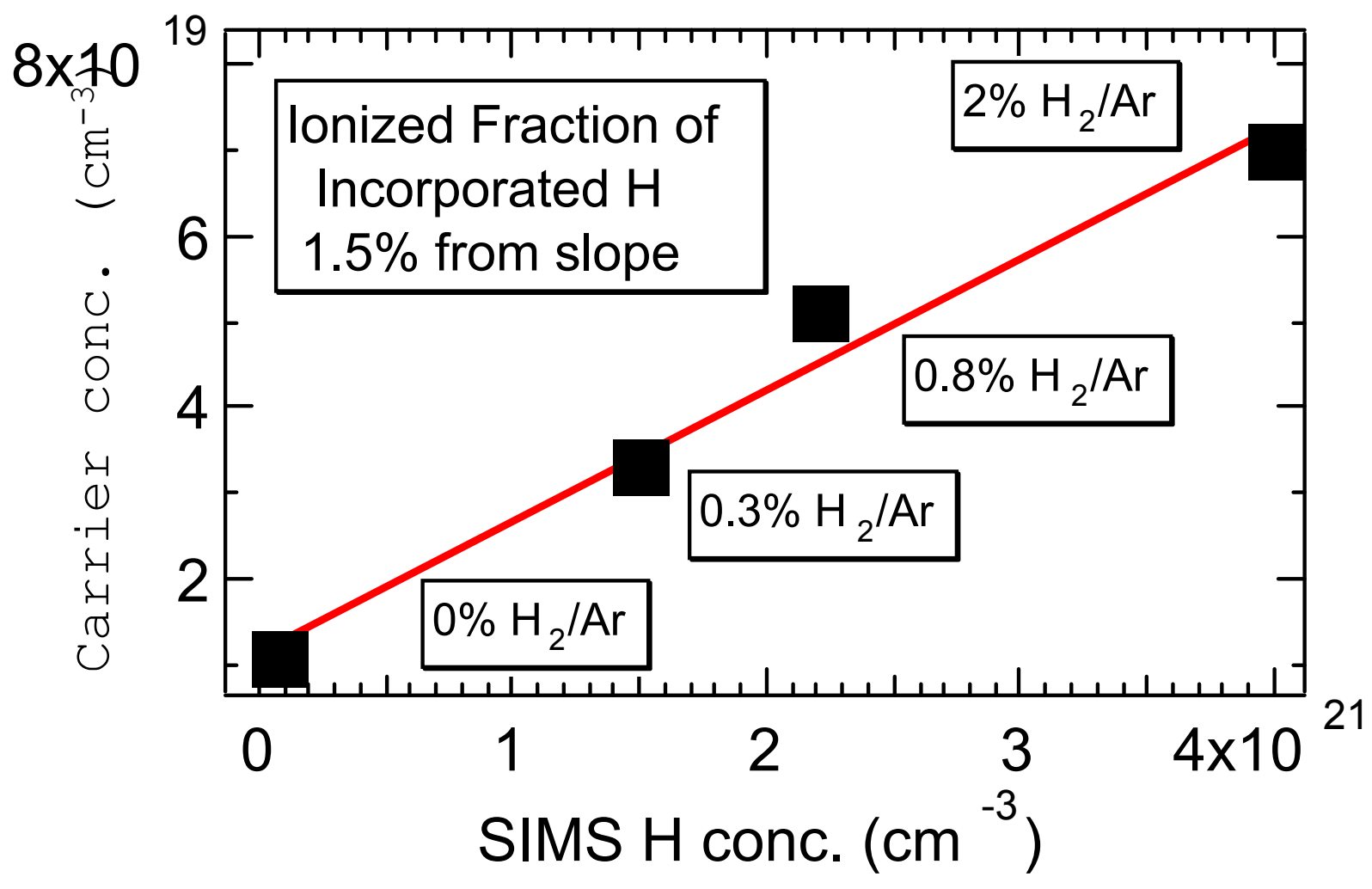

SIMS measurements by Matthew R. Young, NREL 


\section{At what $\mathrm{T}$ is $\mathrm{H}_{2}$ removed from $\mathrm{ZnO}$ ?}

Undoped $\mathrm{ZnO}, 0.3 \% \mathrm{H}_{2} / \mathrm{Ar}$ Annealed $1 \mathrm{hr}$. at each temp. Dep. Temp. $200^{\circ} \mathrm{C}$

- $\operatorname{Ar}$ $\bowtie \mathrm{N}_{2}$

Dep. Temp. $25^{\circ} \mathrm{C}$

$\triangle \mathrm{Ar}$
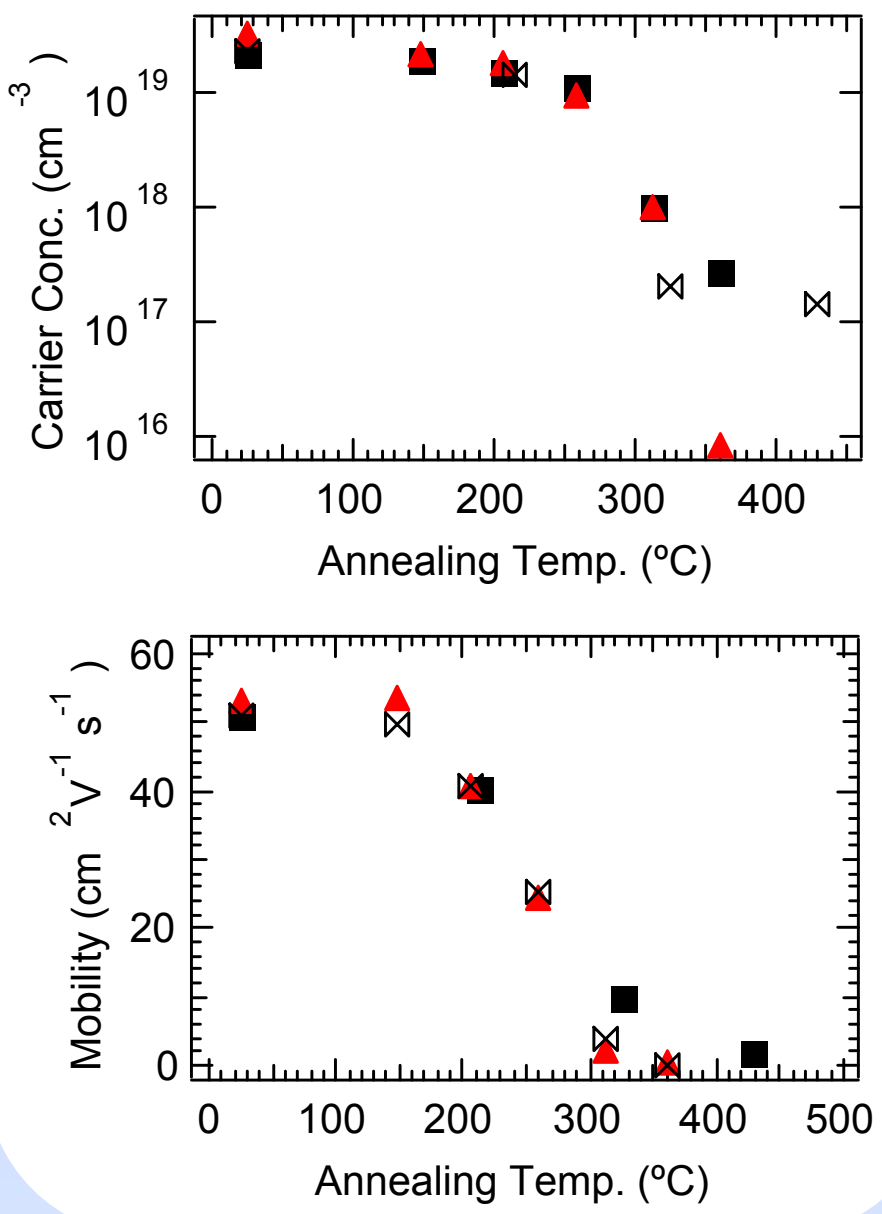

- Decrease in carrier concentration and mobility appears near temp. at which desorption occurs

\section{Temperature-Programmed Desorption}

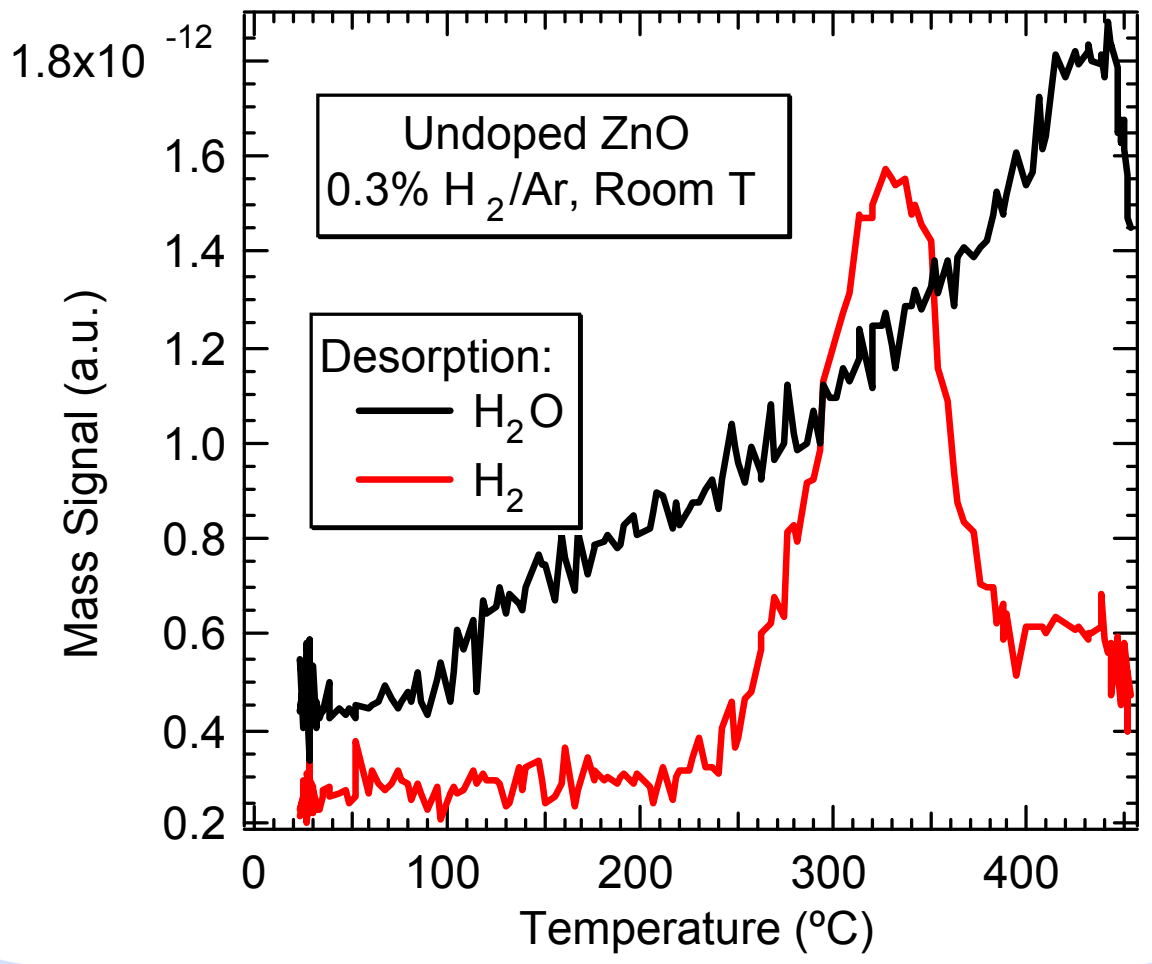

Measurement performed by Anne Dillon, NREL 


\section{Structure $-\mathrm{H}_{2}$ and Thickness effects}

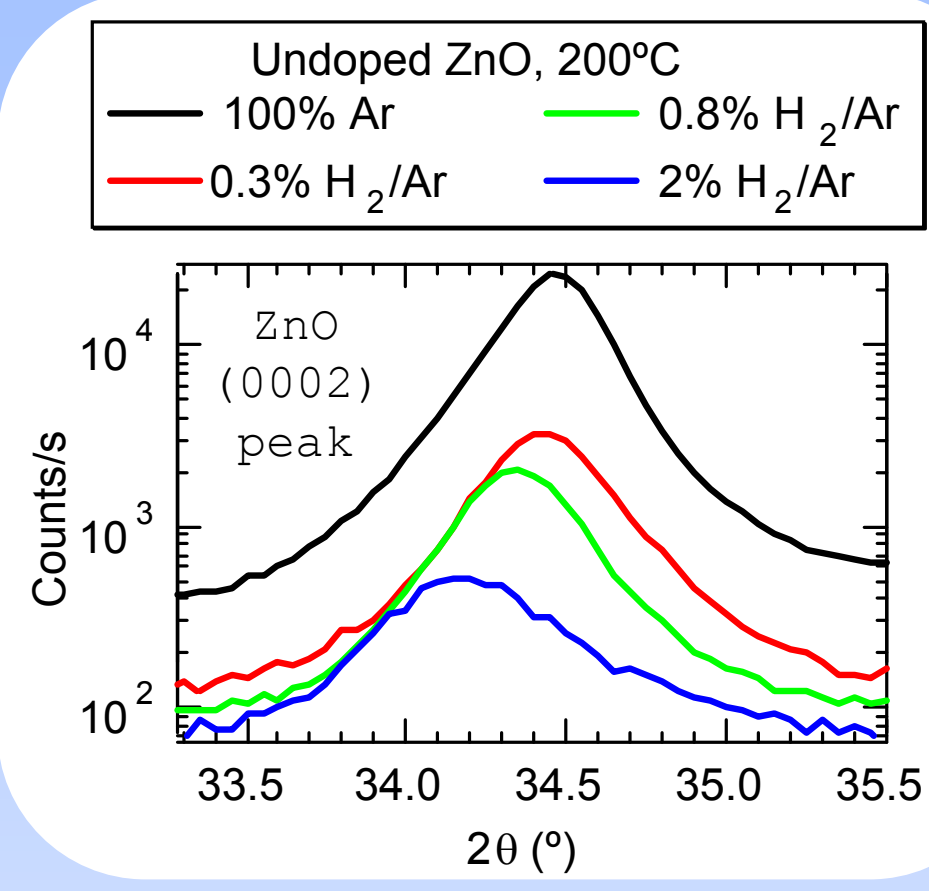

- Peak shifts to lower angle and decreases in intensity with $\mathrm{H}_{2} / \mathrm{Ar}$

- But film thickness also decreases by up to $50 \%$ with ornixth in $\mathrm{H}$

$\mathrm{ZnO}$ film lattice spacing, substrate temp. $200^{\circ} \mathrm{C}$

$\rightarrow-\mathrm{ZnO}$ undoped

$\rightarrow$ ZnO:Al (0.1 wt.\%)

- ZnO:Al (0.2 wt.\%)

$\neg \mathrm{ZnO}: \mathrm{Al}(0.5 \mathrm{wt} . \%)$

$\rightarrow$ ZnO:Al (1 wt.\%)

$\longrightarrow$ ZnO:Al (2 wt.\%)

-.. ZnO (0002) (JCPDS 36-1451)

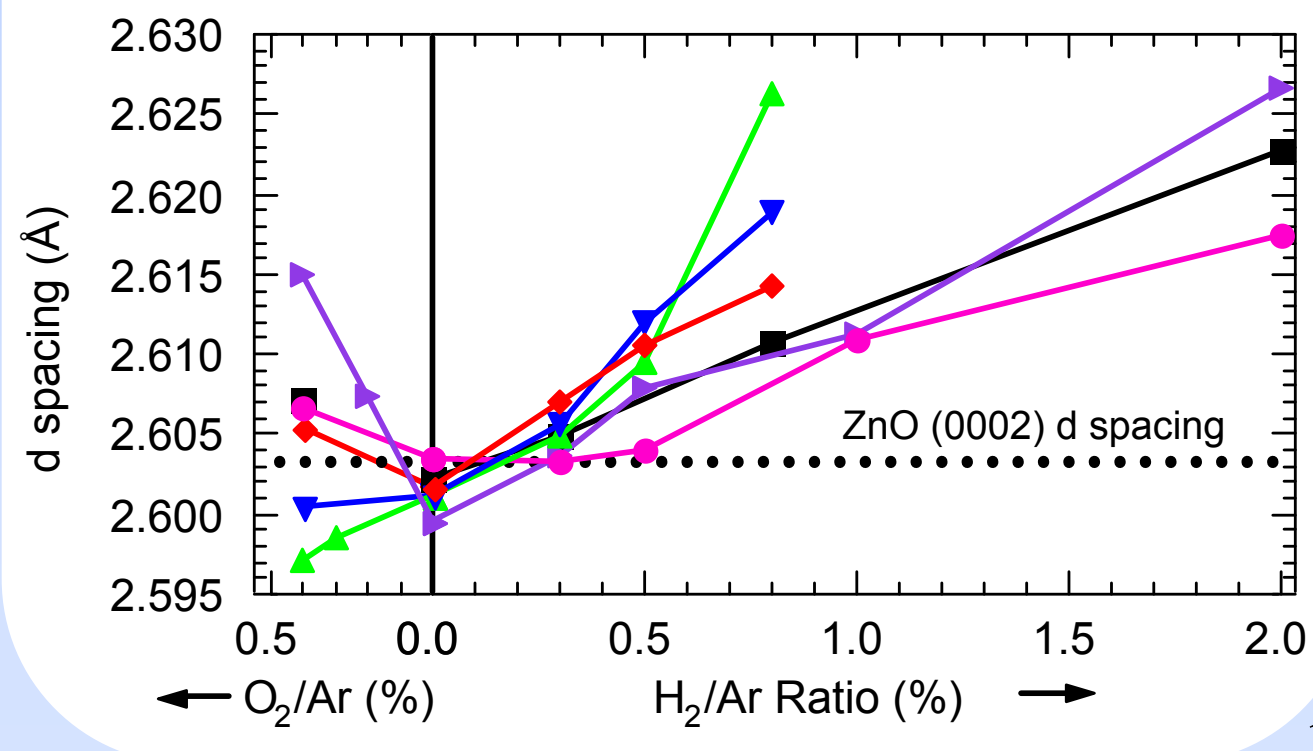

$\leftarrow \mathrm{O}_{2} / \mathrm{Ar}(\%)$

- Is change in $\mathrm{d}$ spacing due to $\mathrm{H}_{2}$ or thickness?

- To what extent is $\mathrm{H}_{2}$ incorporated into films? 


\section{Separating $\mathrm{H}_{2}$ and Thickness Effects}

- Empirical fit of d spacing vs.

ZnO:Al (0.2 wt.\%) Room T, 0.3\% H ${ }_{2} / \mathrm{Ar}$

-. ZnO (0002) bulk

— Fit to d spacing

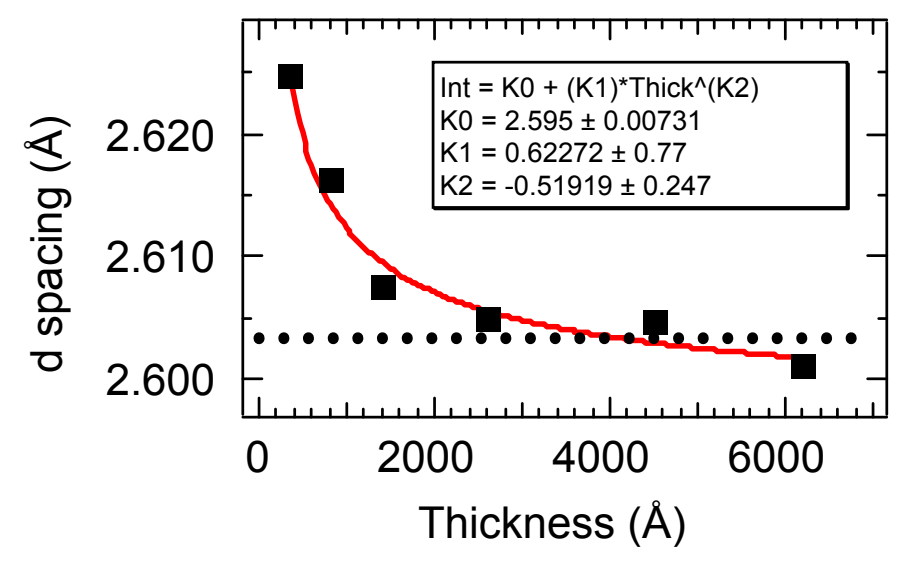

\begin{tabular}{|lll|}
\hline Undoped ZnO & $\diamond$ & ZnO:Al (0.5 wt.\%) \\
ZnO:Al (0.1 wt.\%) & $\nabla$ & ZnO:Al (1 wt.\%) \\
ZnO:Al (0.2 wt.\%) & $\triangle$ & ZnO:Al (2 wt.\%) \\
\hline
\end{tabular}

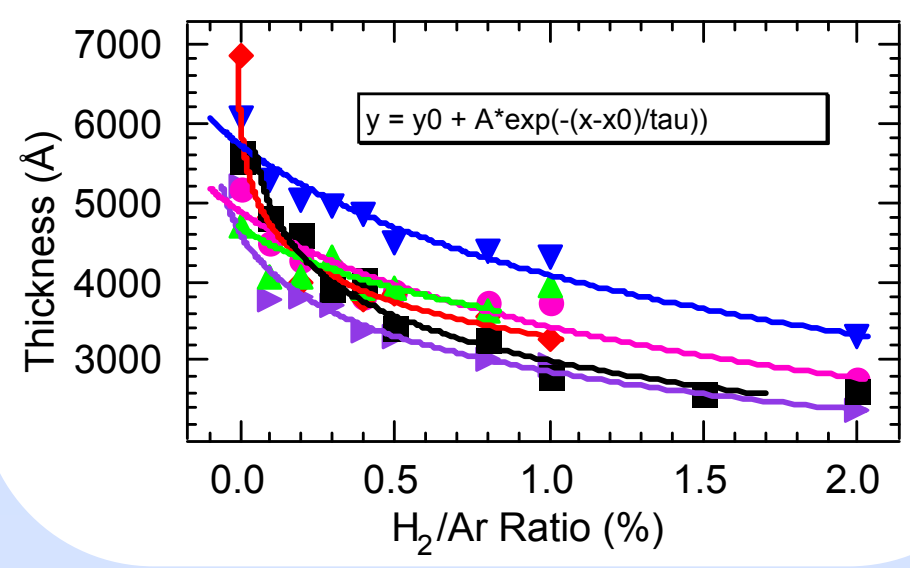
thickness for fixed $\mathrm{Al}$ and $\mathrm{H}_{2}$ amounts

- Fit of $\mathrm{H}_{2}$ vs. thickness for all Al amounts

\begin{tabular}{|c|}
\hline ZnO film lattice spacing, substrate temp. $200^{\circ} \mathrm{C}$ \\
- ZnO undoped
\end{tabular}

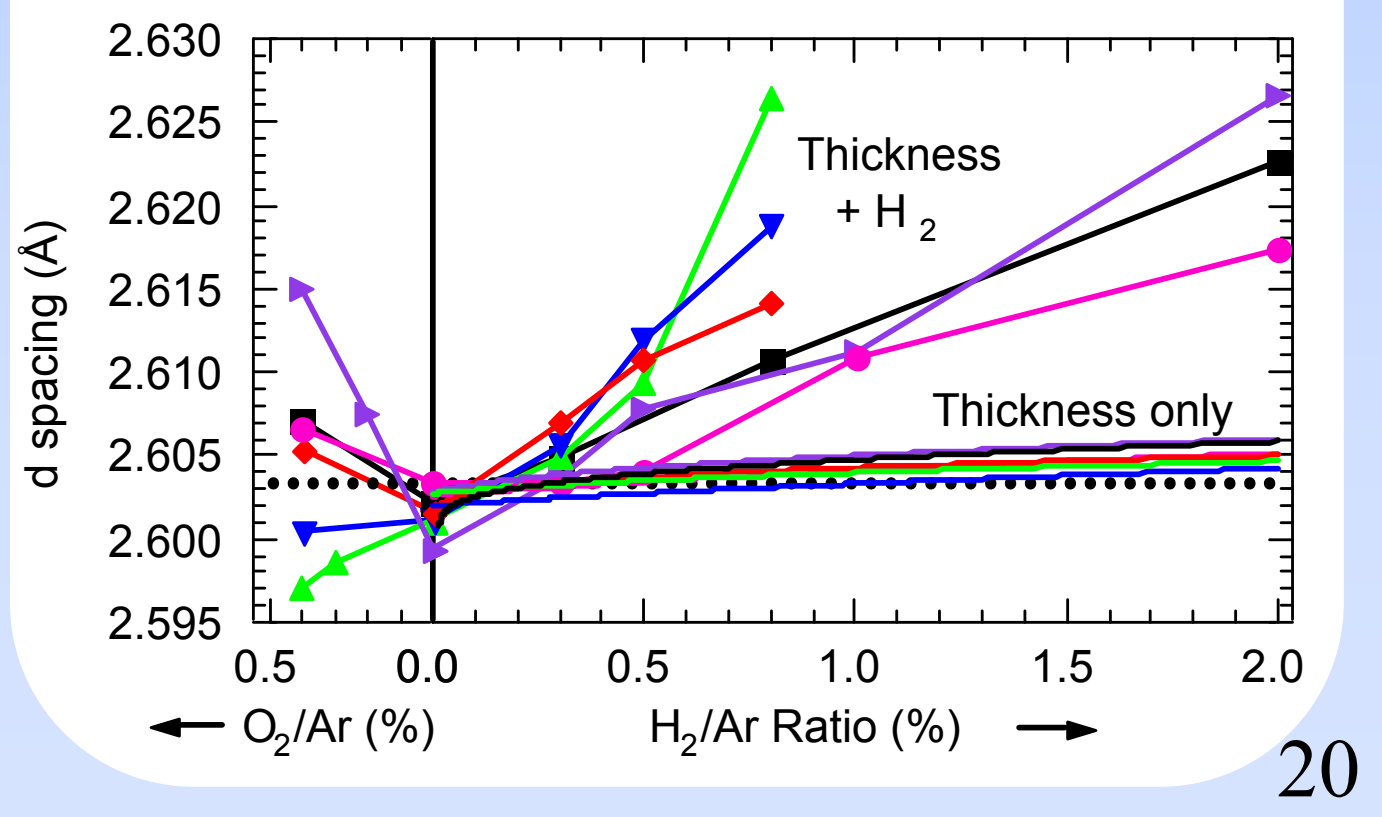




\section{Scattering Mechanisms Using T-dep. Hall}

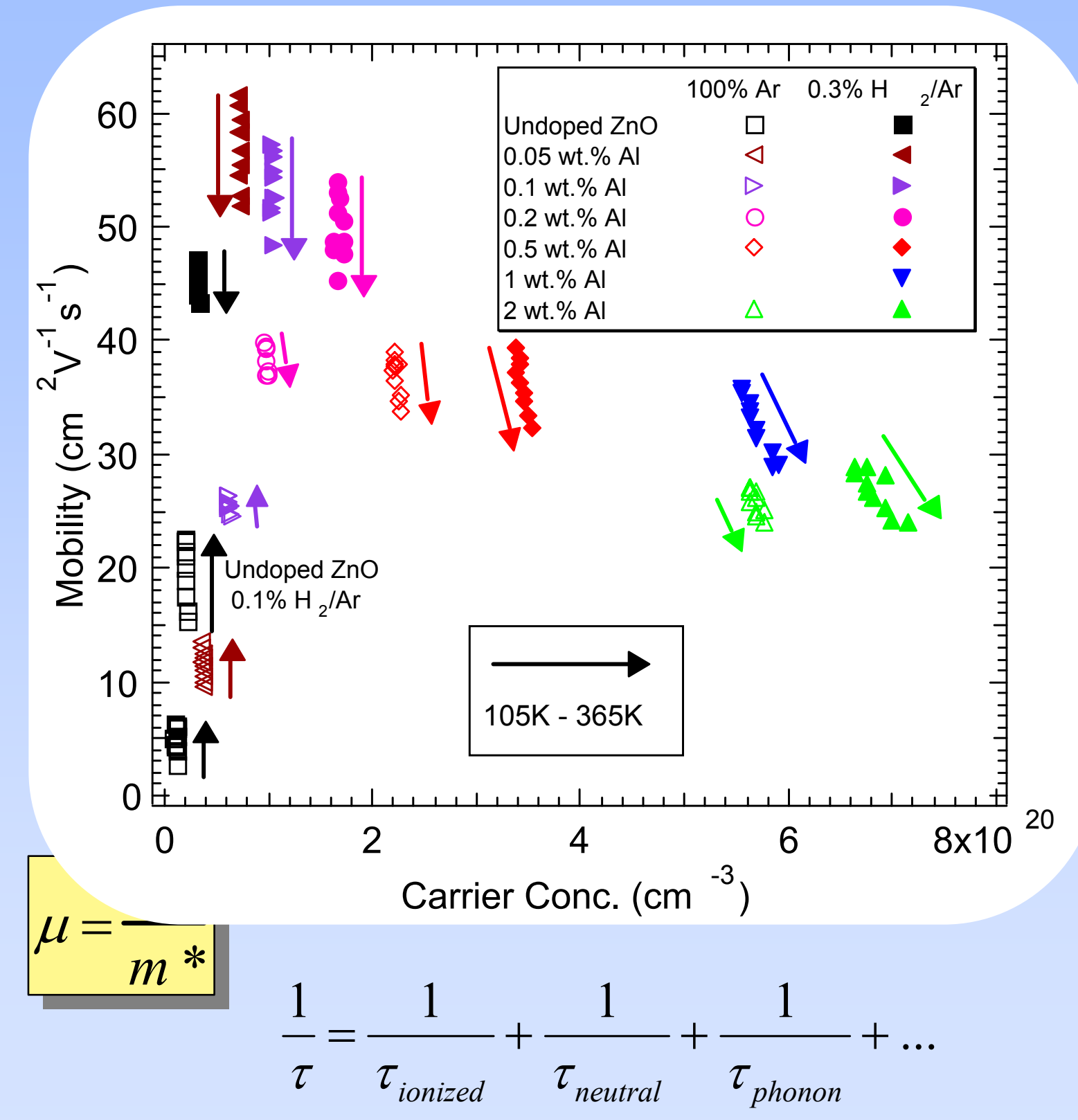

Undoped $\mathrm{ZnO}$

$0.1 \% \mathrm{H}_{2} / \mathrm{Ar}$

- Temp. activation $\Rightarrow$ barrier (dangling bonds?)

$0.3 \% \mathrm{H}_{2} / \mathrm{Ar}$

- Phonon scattering

- Passivation of dangling bonds at grain boundaries

$\mathrm{ZnO}: \mathrm{Al}$

- Increasing ionized impurity scattering with Al dopant 


\section{Dopant Ionization - EPMA}

Substrate temp. $200^{\circ} \mathrm{C}$

$\multimap$ ZnO:Al (0.05 wt.\%)

$\rightarrow$ ZnO:Al (0.1 wt.\%)

$\multimap$ ZnO:Al (0.5 wt.\%)

$\rightarrow$ ZnO:Al (1.0 wt.\%)

- ZnO:Al (2.0 wt.\%)

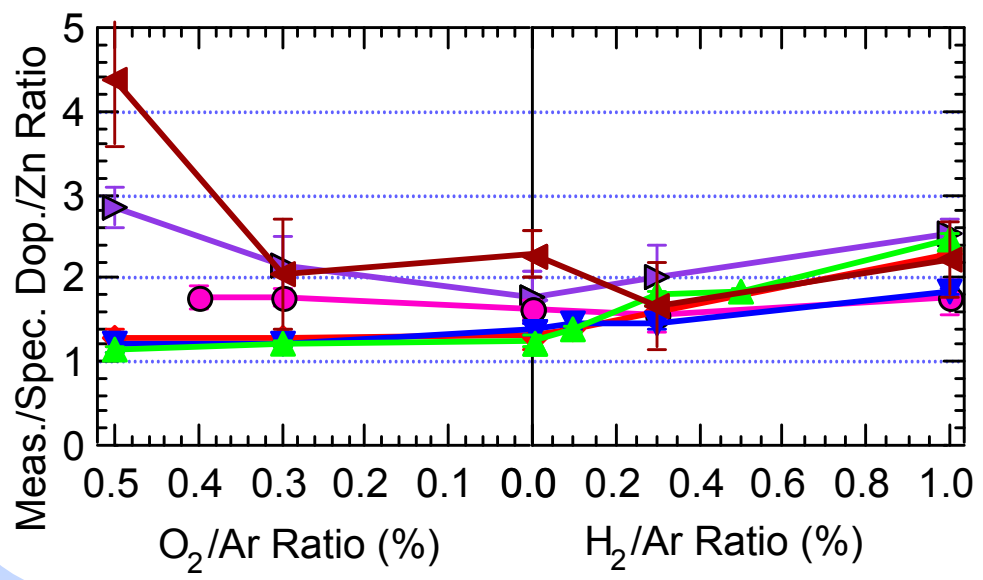

Ionization $\%=\frac{n_{\text {Doped }}-n_{\text {Undoped ZnO }}}{n_{\mathrm{E}}}$

$$
n_{\text {EPMA }}
$$

- Limited $\mathrm{H}_{2}$ aids ionization

- Ionization decreases with Al level

- Mo has poorest ionization

Measurements performed by Bobby To, NREL
- Mo-doped films contain near the amount of dopant specified

- Al-doped films all contain greater amts. of $\mathrm{Al}$
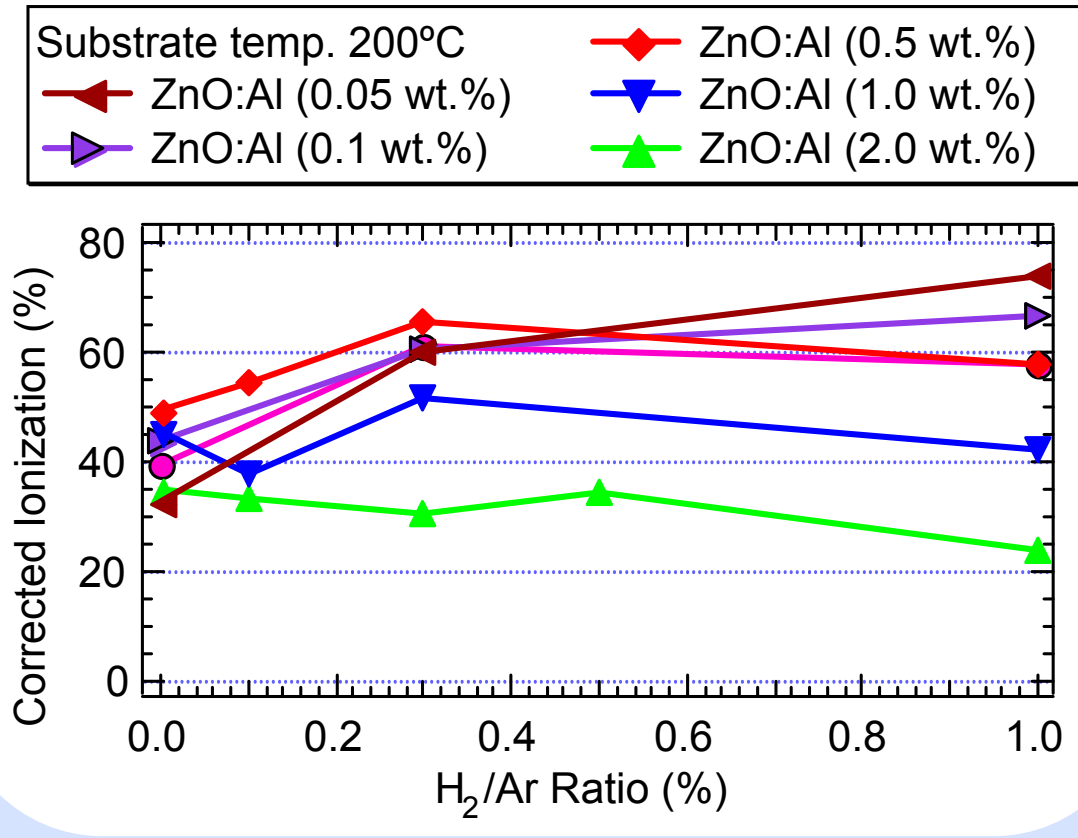


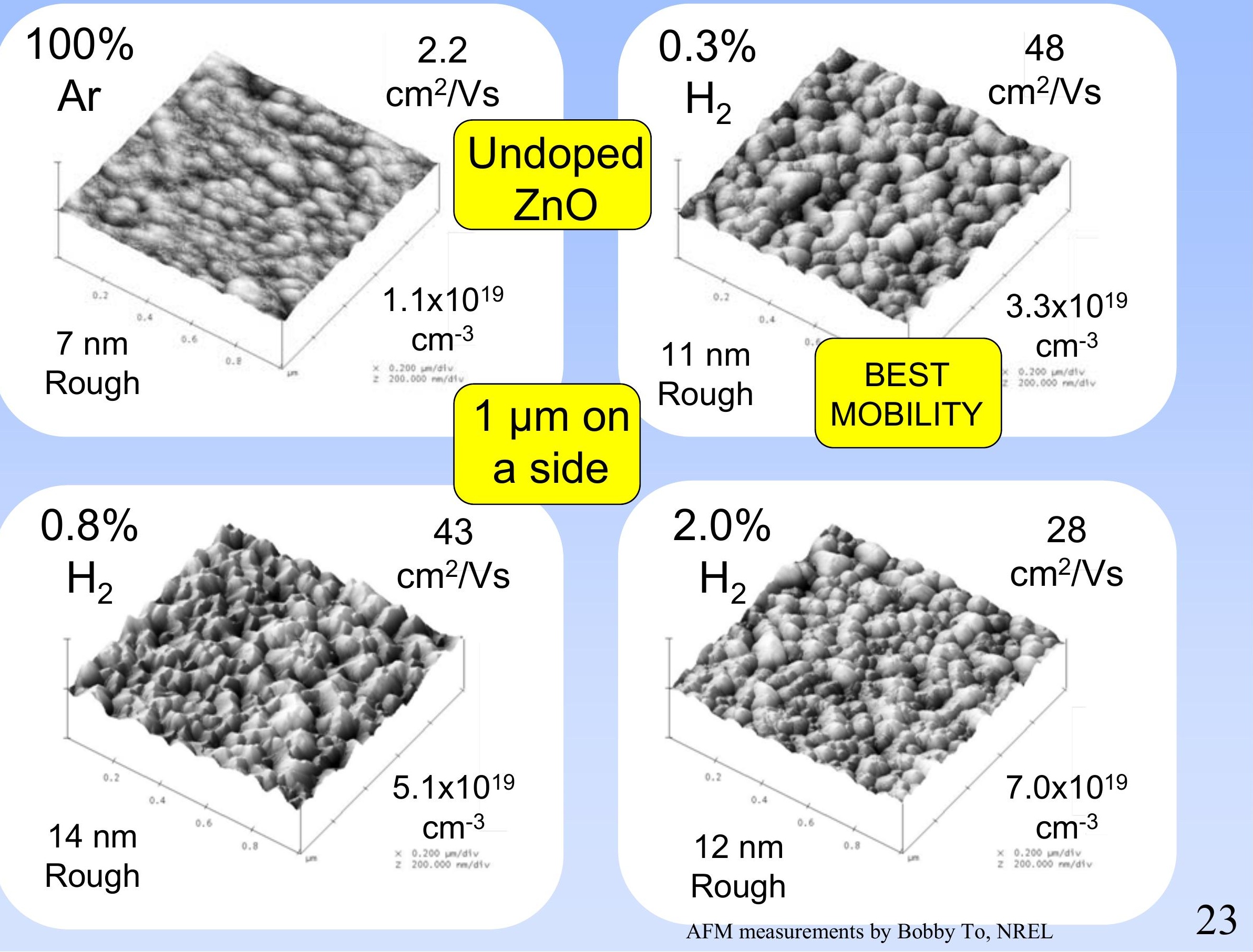




\section{Film Structure from SEM}

$0.1 \% \mathrm{Al}_{2} \mathrm{O}_{3}$ $200^{\circ} \mathrm{C}$

\section{$0.3 \% \mathrm{O}_{2} / \mathrm{Ar}$}
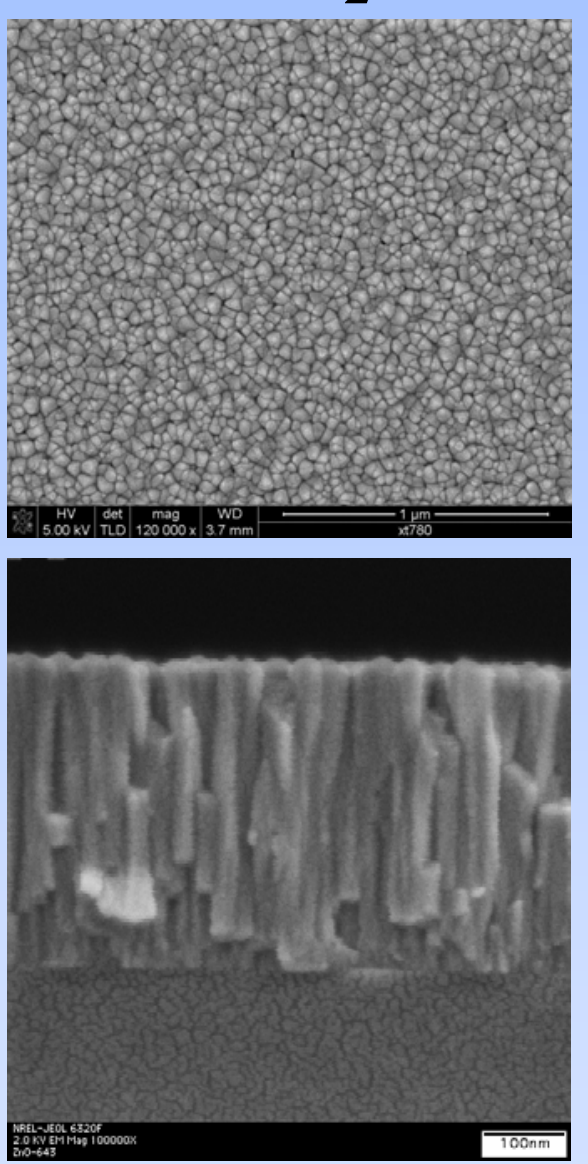

Scales

Top: $2.1 \mu \mathrm{m}$ wide Bottom: $0.73 \mu \mathrm{m}$ wide
$100 \% \mathrm{Ar}$

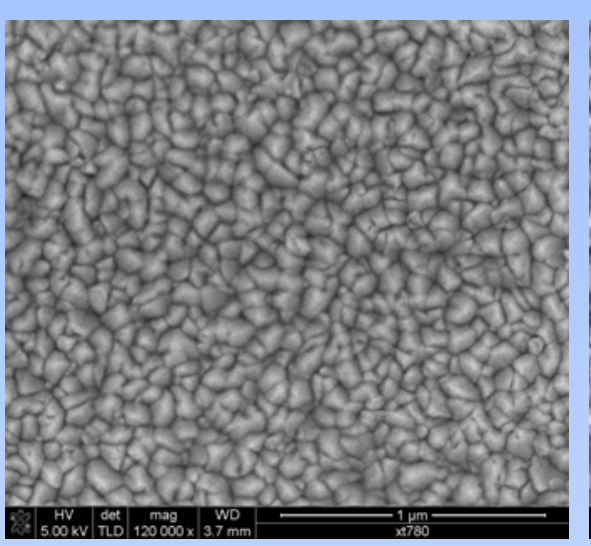

$0.4 \% \mathrm{H}_{2} / \mathrm{Ar}$
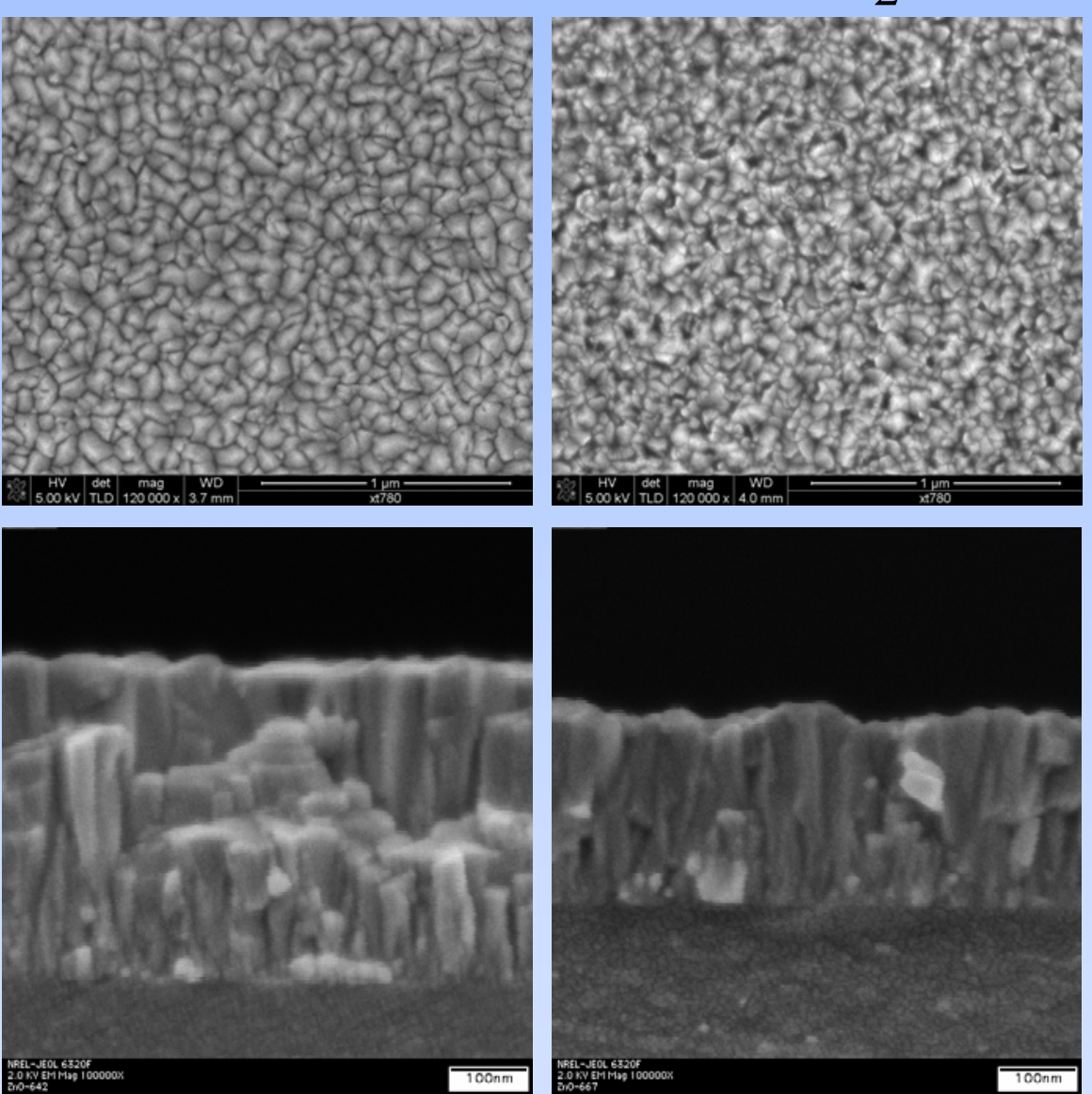

Increasing roughness and faceting

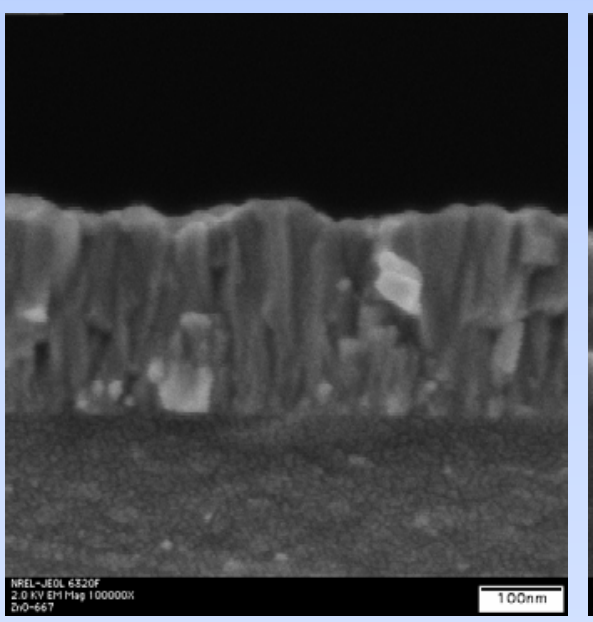

$1.0 \% \mathrm{H}_{2} / \mathrm{Ar}$
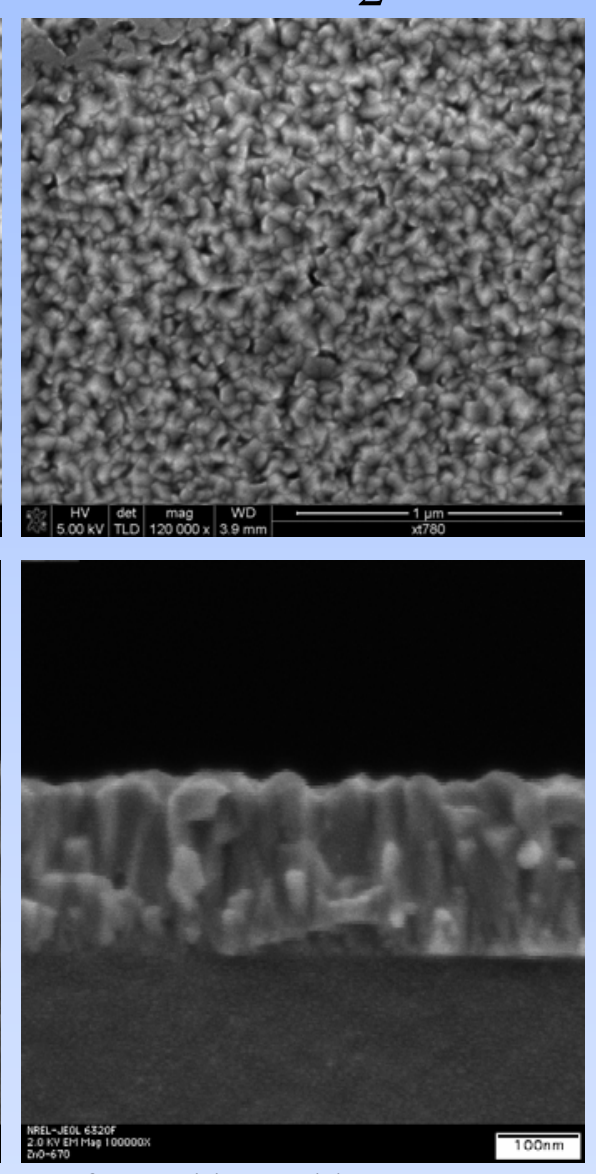

Performed by Bobby To, NREL Increasing lateral crystallite growth Does lateral growth improve electrical properties? 


\section{Native Defects: Why is Undoped $\mathrm{ZnO}$ n-type?}

- Oxygen vacancies? ${ }^{1-3}$

- High formation energy, deep donor $^{4}$

- Zn interstitials? ${ }^{5}$

- High formation energy, high diffusivity ${ }^{4}$

- Hydrogen as dopant (bonded to $\mathrm{O}$ )

$-\mathrm{H}_{\text {interstitial }}{ }^{6}$

$-\mathrm{H}_{2}$ in $\mathrm{Zn}$ vacancy ${ }^{7}$

- $\mathrm{H}$ always a donor in $\mathrm{ZnO}^{8-11}$

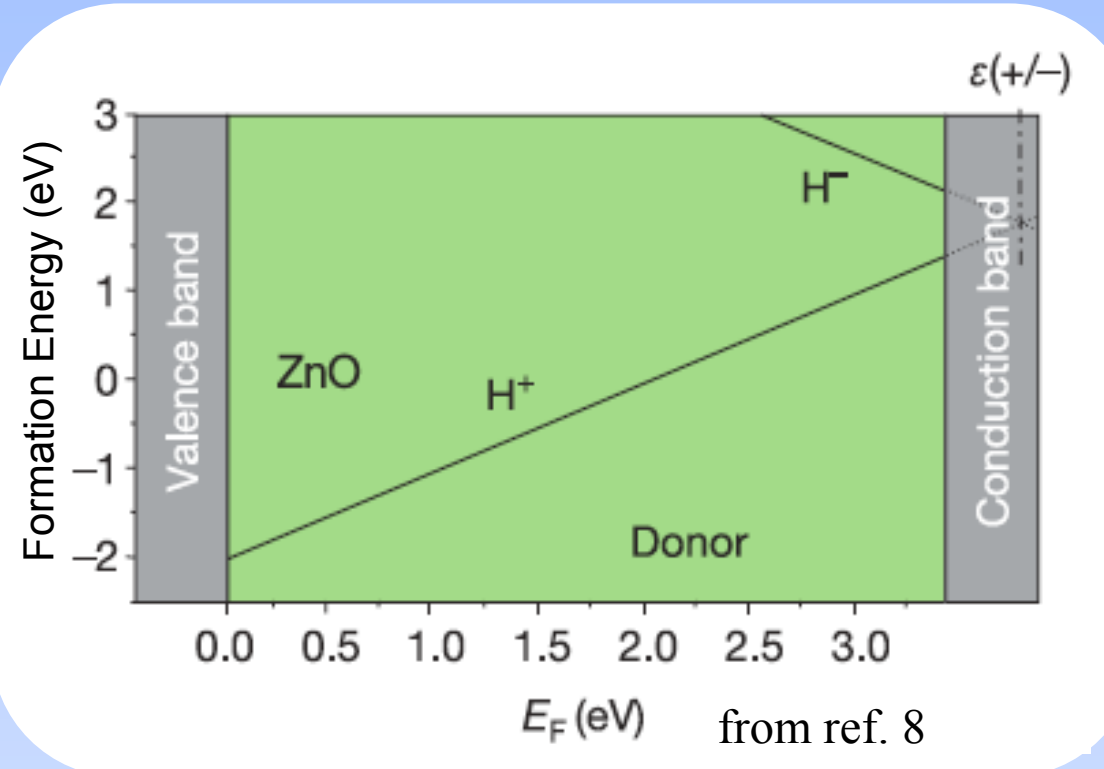

${ }^{1}$ G.D. Mahan, J. Appl. Phys. 54, 3825 (1983).

${ }^{2}$ E. Ziegler et. al., Phys. Status Solidi A 66, 635 (1981).

${ }^{3}$ A.F. Kohan et. al., Phys. Rev. B 61, 15019 (2000).

${ }^{4}$ A. Janotti and C.G. Van de Walle, J. Crys. Growth 287, 58 (2006).

${ }^{5}$ D.C. Look et. al., Phys. Rev. Lett. 82, 2552 (1999).

${ }^{6}$ C.G. Van de Walle, Phys. Rev. Lett. 85, 1012 (2000).

${ }^{7}$ E. V. Lavrov et. al., Phys. Rev. B 66, 165205 (2002).

${ }^{8}$ C.G. Van de Walle and J. Neugebauer, Nature 423, 626 (2003).

${ }^{9}$ C.G. Van de Walle, Phys. Stat. Sol. B 235, 89 (2003).

${ }^{10}$ Ç. Kiliç and A. Zunger, Appl. Phys. Lett. 81, 73 (2002).

${ }^{11}$ A. Janotti and C.G. Van de Walle, Nature Materials 6, 44 (2007). 


\section{Benefits of $\mathrm{ZnO}$ TCO}

- May be less expensive than comparable materials (e.g. ITO)

- No adverse effects from $\mathrm{H}_{2}$-rich plasma

- High transparency in visible and near-IR

Single-junction Multi-junction

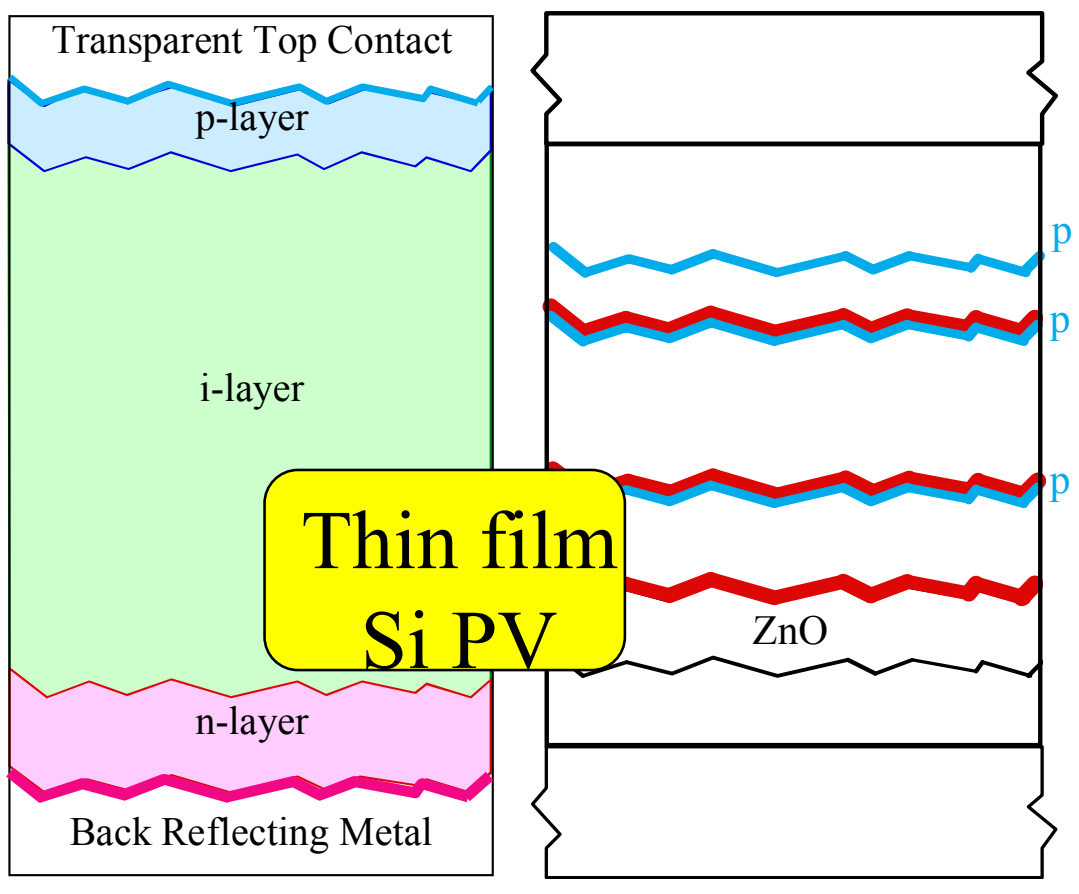

Brent Nelson, NREL

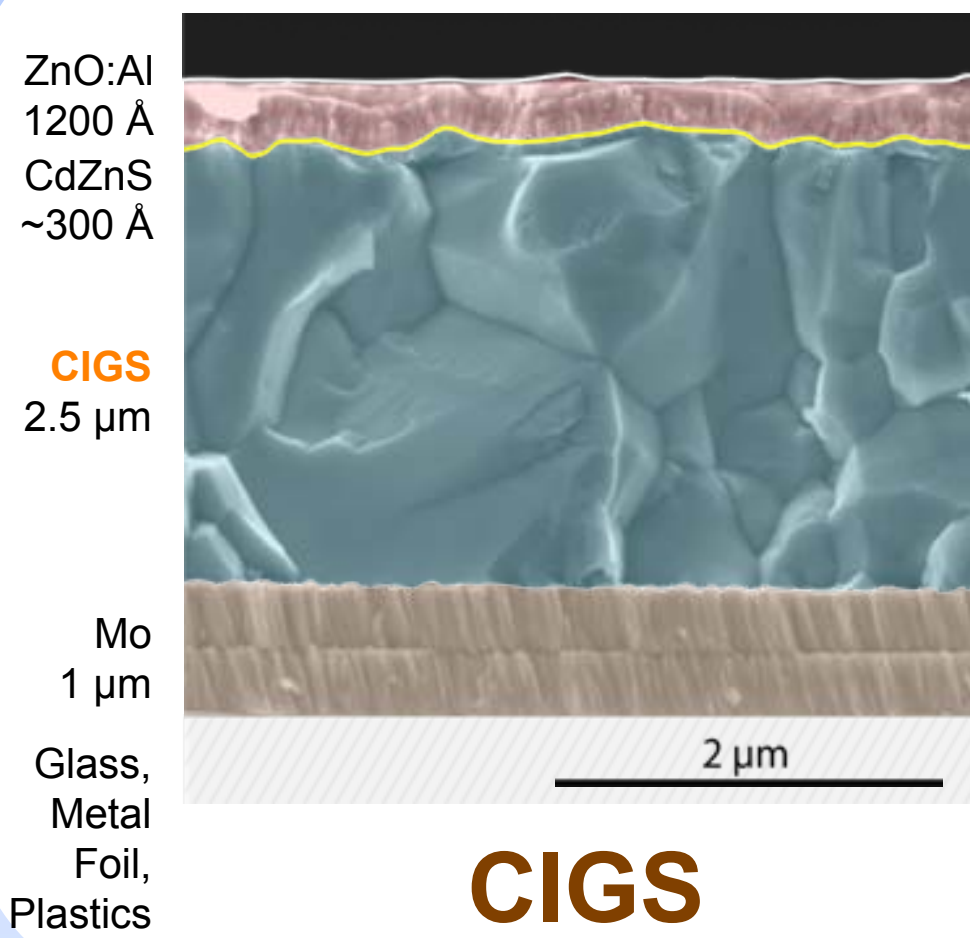

R. Noufi, NREL 